OPEN ACCESS

Edited by:

Kai Wang,

Chinese Academy of Agricultural

Sciences (CAAS), China

Reviewed by:

Xuepeng Chi,

Shandong Agricultural University,

China

Miao Long,

Shenyang Agricultural University,

China

Xi Ma,

China Agricultural University, China

*Correspondence:

Lingna Zhang

zhanglingna1043@outlook.com

Baichuan Deng

dengbaichuan@scau.edu.cn

${ }^{+}$These authors have contributed equally to this work

Specialty section:

This article was submitted to Nutritional Immunology,

a section of the journal

Frontiers in Immunology

Received: 12 November 2021 Accepted: 21 December 2021

Published: 14 January 2022

Citation:

Yang K, Deng $X$, Jian S, Zhang M, Wen $C$, Xin Z, Zhang L, Tong A, Ye S, Liao P, Xiao Z, He S, Zhang F, Deng J, Zhang L and Deng B (2022) Gallic Acid

Alleviates Gut Dysfunction and

Boosts Immune and Antioxidant

Activities in Puppies Under

Environmental Stress Based on Microbiome-Metabolomics Analysis.

Front. Immunol. 12:813890.

doi: 10.3389/fimmu.2021.813890

\section{Gallic Acid Alleviates Gut Dysfunction and Boosts Immune and Antioxidant Activities in Puppies Under Environmental Stress Based on Microbiome-Metabolomics Analysis}

\author{
Kang Yang ${ }^{1+}$, Xiaolin Deng ${ }^{2 \dagger}$, Shiyan Jian ${ }^{1}$, Meiyu Zhang ${ }^{3}$, Chaoyu Wen ${ }^{1}$, \\ Zhongquan Xin ${ }^{1}$, Limeng Zhang ${ }^{1}$, Aorigeile Tong ${ }^{4}$, Shibin $Y^{1}{ }^{1}$, Pinfeng Liao ${ }^{1}$, Zaili Xiao ${ }^{1}$, \\ Shansong $\mathrm{He}^{1}$, Fan Zhang ${ }^{1}$, Jinping Deng ${ }^{1}$, Lingna Zhang ${ }^{1 *}$ and Baichuan Deng ${ }^{1 *}$

\begin{abstract}
1 Guangdong Laboratory for Lingnan Modern Agriculture, Guangdong Provincial Key Laboratory of Animal Nutrition Control, National Engineering Research Center for Breeding Swine Industry, College of Animal Science, South China Agricultural University, Guangzhou, China, ${ }^{2}$ Department of Urology, Ganzhou People's Hospital, Ganzhou, China, ${ }^{3}$ College of Animal Nutrition, Guangzhou Qingke Biotechnology Co., Ltd., Guangzhou, China
\end{abstract} \\ Science and Technology, Guangdong Polytechnic of Science and Trade, Guangzhou, China, ${ }^{4}$ Research Center of Pet
}

Early-life exposure to environmental stress disrupts the gut barrier and leads to inflammatory responses and changes in gut microbiota composition. Gallic acid (GA), a natural plant polyphenol, has received significant interest for its antioxidant, antiinflammatory, and antimicrobial properties that support the maintenance of intestinal health. To assess whether dietary supplementation of GA alleviates environmental stress, a total of 19 puppies were randomly allocated to the following three dietary treatments for 2 weeks: 1) basal diet (control (CON)); 2) basal diet + transportation (TS); and 3) basal diet with the addition of $500 \mathrm{mg} / \mathrm{kg}$ of $\mathrm{GA}+$ transportation (TS+GA). After a 1-week supplementation period, puppies in the TS and TS+GA groups were transported from a stressful environment to another livable location, and puppies in the CON group were then left in the stressful environment. Results indicated that GA markedly reduced the diarrhea rate in puppies throughout the trial period and caused a moderate decline of serum cortisol and HSP-70 levels after transportation. Also, GA alleviated the oxidative stress and inflammatory response caused by multiple environmental stressors. Meanwhile, puppies fed GA had a higher abundance of fecal Firmicutes and Lactobacillus and lower Proteobacteria, Escherichia-Shigella, and Clostridium_ sensu_stricto_1 after transportation. As a result, the TS+GA group had the highest total short-chain fatty acids and acetic acid. Also, the fecal and serum metabolomics analyses revealed that GA markedly reversed the abnormalities of amino acid metabolism, lipid metabolism, carbohydrate metabolism, and nucleotide metabolism caused by stresses. Finally, Spearman's correlation analysis was carried out to explore the 
comprehensive microbiota and metabolite relationships. Overall, dietary supplementation of GA alleviates oxidative stress and inflammatory response in stressed puppies by causing beneficial shifts on gut microbiota and metabolites that may support gut and host health.

Keywords: environmental stress, gallic acid, puppy, antioxidant, inflammatory response, microbiome, metabolomics

\section{INTRODUCTION}

Stress response is a ubiquitous physiological response elicited when the threat to the homeostasis is perceived by the organism due to environmental, physical, or psychological stimuli (1). Early-life exposure to a specific environment can influence the development and function of multiple organs and systems, including the central nervous, gastrointestinal, and immune systems (2-4). Current evidence suggests that the hypothalamic-pituitary-adrenal (HPA) axis is the major pathway that controls the production of the stress hormones, glucocorticoids (GC) in response to various environmental factors (e.g., oxidative stress, heat, and osmotic stress). A series of metabolic and immune-suppressive effects (5) are elicited by GC, acting through the glucocorticoid receptor. Specifically, the HPA axis is activated by the secretion of corticotropin-releasing hormone $(\mathrm{CRH})$ from the hypothalamus, which induces the anterior pituitary gland to release adrenocorticotropic hormone $(\mathrm{ACTH})$, and then ACTH stimulates the adrenal cortex to release the GC, mainly cortisol (COR), which negatively regulates $\mathrm{CRH}$ production to terminate the stress response cascade (6-8). Moreover, heat shock proteins (HSPs), a kind of stress-induced proteins ubiquitously found in germs and mammals (9-11), are heavily involved in dealing with environmental stress (12). Particularly, HSP-70 serves as a molecular chaperone to protect cells against the stresses of various types and origins. A recent study has demonstrated that HSP-70 helps to maintain and stabilize the intestinal tight junctions, as a result generating a stronger intestinal barrier in the ileum of stressed animals (13, 14). Simultaneously, environmental stressors trigger the production of intracellular reactive oxygen species (ROS) that can disrupt the cellular antioxidant defense system (15). Stressinduced production of ROS may be mediated by the inflammatory response because inflammation is associated with high levels of ROS, and strong stressors can induce an inflammatory response (16).

Stress not only affects the physiological and stress system but also destroys gut microbiota (GM) (17-19). The human body is inhabited by trillions of microorganisms that participate in nutrient metabolism and influence the health and immune responses of the host (20-22). Lactobacillus and Bifidobacterium are the main genera of probiotic bacteria, which enhance the host immune system and favorably modulate gastrointestinal physiology $(23,24)$. Moreover, the producers of short-chain fatty acids (SCFAs), the phylum Firmicutes and the genera Faecalibacterium and Roseburia, may also be considered beneficial bacteria (25-28) because SCFAs are a carbon energy source for intestinal epithelial cells and can induce the development of intestinal Treg cell with potent anti-inflammatory functions (29-31). Conversely, the pathogenic bacteria Enterobacteriaceae (belong to the phylum Proteobacteria), a family including Escherichia, Shigella, Proteus, and Klebsiella, is often associated with the development of systemic inflammation $(32,33)$. It is increasingly recognized that the acute and chronic stressors that activate the HPA axis can modulate GM and may be one causal factor in gut dysbiosis (1). In support, recent evidence has begun to connect GM and its metabolites to gastrointestinal diseases, inflammation, and psychological metrics in humans suffering from multiple stressors $(8,17,18)$. Collectively, these studies provide preliminary evidence that GM may respond to environmental stress.

Polyphenol performs antioxidant and anti-inflammatory properties and can modulate oxidative stress and inflammatory signaling (34-36). Growing evidence indicates that polyphenol contributes to gut health via the modulation of colon microbiota composition (37-39). Gallic acid (GA), also known as 3,4,5trihydroxybenzoic acid, is a natural polyphenol compound present in fruits, vegetables, and herbal medicines (40). It has been reported that GA effectively inhibited inflammation $(41,42)$ and oxidation $(43,44)$ in vitro and in vivo and altered metabolic and bacterial profiles in the colitis model (45). As far as we know, there is little discussion about whether GA can relieve the damage caused by multiple stressors. Based on previous research, we hypothesize that multiple stressors can cause inflammation and oxidative stress by promoting the growth of pathogenic bacteria species, thereby causing diarrhea; and dietary supplementation of GA may have a role in alleviating these symptoms.

Beagle dogs are considered excellent models for human microbiome research because of the high similarities in structures and functions between dog and human microbiomes (46). To determine whether changing environment and adding GA are efficacious in preventing the deleterious effects of stress on antioxidative and immune system activity, we transported puppies from a stressful environment to a livable environment. In detail, we evaluated the diarrhea rate, physiological stress, antioxidant capacity, inflammatory response, and metabolites by dietary supplementation of GA at $500 \mathrm{mg} / \mathrm{kg}$ before and after transportation. In parallel, the $16 \mathrm{~S}$ rRNA gene sequencing was adopted to monitor microbiota alterations, and untargeted metabolomics based on ultra-performance liquid chromatography-Orbitrap-tandem mass spectrometry (UPLCOrbitrap-MS/MS) analysis method was employed to capture changes in different metabolic pathways and potential metabolic biomarkers. 


\section{MATERIALS AND METHODS}

\section{Animals and Diet}

All experimental procedures were authorized by the Experimental Animal Ethics Committee of South China Agricultural University (Approval number: 2019188) and were performed following the guidelines of the Laboratory Animal Center at the South China Agricultural University. Animal welfare was monitored by research and animal care staff daily.

A total of 19 beagle dogs (Table 1) were selected in this study and were housed individually in pens $(1.35 \times 0.70 \times 0.75 \mathrm{~m}$ kennels) under an indoor relative humidity and temperature of $96 \% \pm 3 \%$ and $29^{\circ} \mathrm{C} \pm 1{ }^{\circ} \mathrm{C}$, respectively (outdoor relative humidity and temperature were $99 \% \pm 1 \%$ and $32^{\circ} \mathrm{C} \pm 2{ }^{\circ} \mathrm{C}$, respectively) at a 12 -h dark-light cycle at the National Canine Laboratory Animal Resource Bank, Guangzhou General Pharmaceutical Research Institute Co., Ltd (Guangzhou, China). All dogs were dewormed and vaccinated, and no drugs (such as antibiotics) that may alter the GM were given 1 month before the experiment. The blood samples were collected for serum biochemistry and blood routine examination 1 day before the trial. All blood routine and serum biochemistry data were within the normal range except for alkaline phosphatase, creatinine, creatine kinase, mean corpuscular hemoglobin, and lymph (Table S1), indicating that puppies under high temperature and high humidity remained in a stressed state.

Ground corn, flour, fish fat, chicken meal, beef powder, fish meal, soybean meal, amino acid, vitamin, and mineral premixes constituted the basal extruded diets. The chemical and energy composition of the basal diet is listed in Table 2. The basal diet meets all the nutrient recommendations by the Association of American Feed Control Officials (AAFCO, 2017) for puppies (48). Dogs were fed $100 \mathrm{~g}$ of diet twice daily (08:00 and 17:00) to meet the required energy needs based on the calculated metabolizable energy content of the basal diet according to the National Research Council (NRC, 2006) (49). They had free access to fresh water ad libitum. GA (purity > 99\%) was purchased from Wufeng Chicheng Biotech Co., Ltd (Yichang, China). The dose of GA supplemented was based on previous studies (50) with minor modifications. After the adaptation period, $500 \mathrm{mg} / \mathrm{kg}$ of GA were mixed with the basal diet and individually dosed for each dog during the trial period. The daily dose of GA was divided and added equally to each of the two planned daily meals.

\section{Experimental Design}

After 4 weeks of adaptation to a basal diet, these puppies were randomly allocated to one of the three dietary treatments: 1)
TABLE 2 | The chemical and energy composition of basal diet tested.

\begin{tabular}{lc}
\hline Items ${ }^{\mathbf{1}}$ & Basal diet $^{\mathbf{2}}$ \\
\hline $\mathrm{DM}(\%)$ & 90.53 \\
OM (\% DM) & 92.84 \\
CP (\% DM) & 23.91 \\
Acid-hydrolyzed fat (\% DM) & 4.56 \\
TDF (\% DM) & 3.95 \\
GE (kJ/g DM) & 17.00 \\
\hline
\end{tabular}

${ }^{1} D M$, dry matter; OM, organic matter; $C P$, crude protein; TDF, total dietary fiber; GE, gross energy.

${ }^{2}$ Extruded diet: corn flour, flour, fish fat, chicken meal, beef powder, imported fish meal, soybean meal, calcium hydrophosphate, calcium chloride, lysine, methionine, vitamin $A$ vitamin $D$, vitamin $E$, copper sulfate, ferrous sulfate, zinc sulfate, and manganese sulfate.

basal diet (control group, CON group), 2) basal diet (transportation stress group, TS group), and 3) basal diet with the addition of $500 \mathrm{mg} / \mathrm{kg}$ of $\mathrm{GA}$ (TS+GA group). The experimental period was 14 days including 7 to 1 days before transportation (BT7-BT1) and 1 to 7 days after transportation (AT1-AT7). Puppies in the TS and TS+GA groups were exposed to the road transportation for $3 \mathrm{~h}$ (from 14:00 to 17:00) at a speed range of $50 \sim 60 \mathrm{~km} / \mathrm{h}$ on day 7 of the experiment in a thermostatic truck at $26^{\circ} \mathrm{C}$ with $50 \%$ in humidity, and no environmental changes we made to the CON group during the study. Thirteen puppies in the TS and TS+GA groups were transported to the Laboratory Animal Center Building at the South China Agricultural University and housed individually in pens $(1.2 \times 1.0 \times 1.1 \mathrm{~m}$ kennels $)$ under a constant temperature and humidity $\left(23^{\circ} \mathrm{C}\right.$ and $70 \%$, respectively) with a light/dark cycle of $12 \mathrm{~h}$. All dogs were continued on their respective diets for another week and given access to toys for behavioral enrichment at all times and to exercise outside of their cages and socialize with each other or humans at least once a day. The study design is depicted in Figure 1.

\section{Chemical Analysis of Diet}

Throughout the trial period, a 200 -g basal diet was collected weekly and was kept in the refrigerator at $-20^{\circ} \mathrm{C}$. Feed samples were dried in the oven and were ground through a 1-mm screen for chemical composition analysis. The dry matter (DM) and organic matter (OM) were determined for the diets according to AOAC (2000; method 950.46 for water and method 942.05 for crude ash) (51). Acid-hydrolyzed fat was analyzed by a fatty analyzer (FT640, Guangzhou, Grand Analytical Instrument Co., Ltd) according to AOAC (2000; method 920.39 for ether extract) (51). The crude protein (CP) was done by using the Kjeldahl method with semi-automatic Kjeldahl apparatus (VAPODEST 200, C. Gerhardt GmbH \& Co. KG, Germany) and following the Official Method of AOAC (2000; method 954.01 for crude

TABLE 1 | Detailed information of beagle dogs in this study.

\begin{tabular}{|c|c|c|c|c|}
\hline Group & Sample size (male:female) & Age (month) & Initial body weight (kg) & $\mathrm{BCS}^{1}$ \\
\hline CON & $6(3: 3)$ & $3.56 \pm 0.32$ & $5.32 \pm 0.91$ & $5.42 \pm 0.49$ \\
\hline TS & $6(2: 4)$ & $3.62 \pm 0.34$ & $5.05 \pm 0.52$ & $5.17 \pm 0.41$ \\
\hline $\mathrm{TS}+\mathrm{GA}$ & $7(3: 4)$ & $3.54 \pm 0.30$ & $5.23 \pm 0.62$ & $5.29 \pm 0.49$ \\
\hline
\end{tabular}

CON, control; TS, transportation; GA, gallic acid.

${ }^{1} B C S$, body condition score; all dogs were weighed, and BCS was assessed using a 9-point scale (47) before morning feeding. Data were expressed as mean \pm SD. 


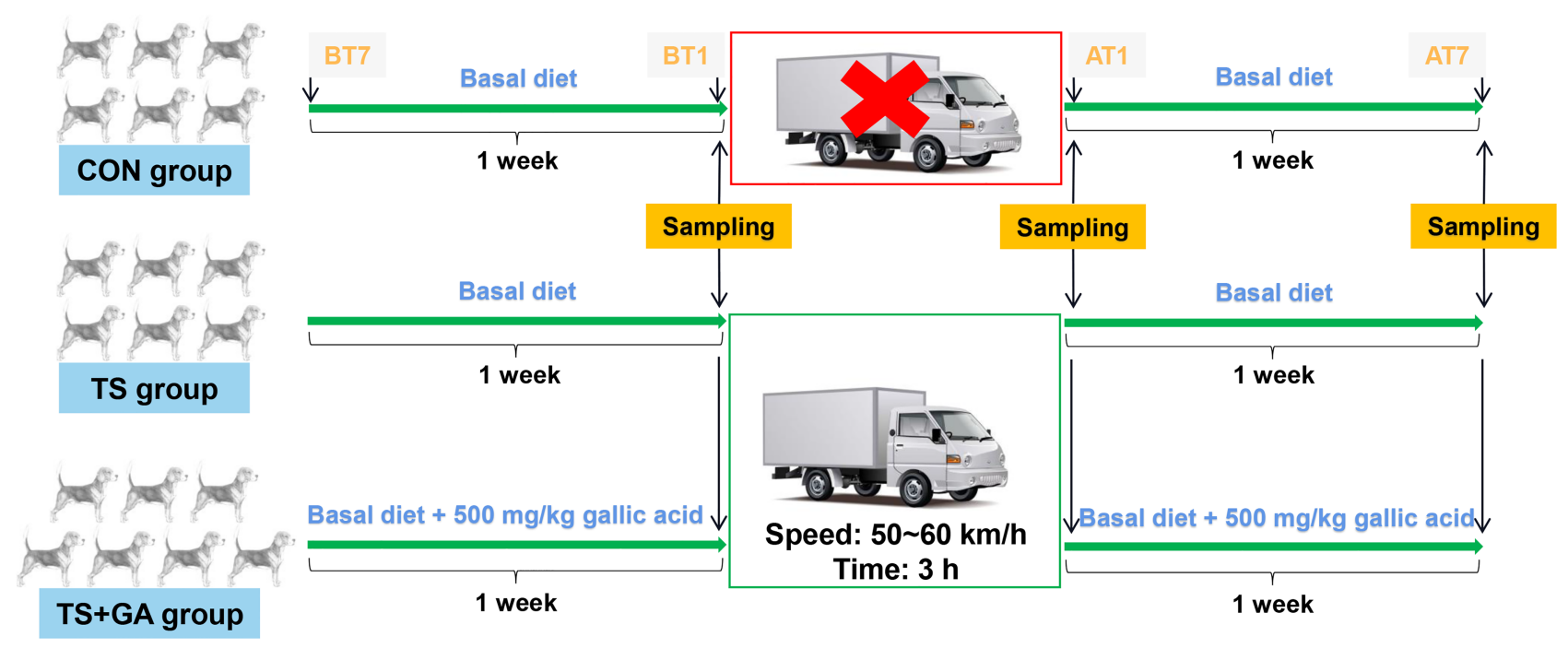

FIGURE 1 | Schematic representation of the study design. BT7, the 7th day before transportation; BT1, the 1st day before transportation; AT1, the 1st day after transportation; AT7, the 7th day after transportation. The CON group was fed basal diet with no transportation ( $\mathrm{n}=6$ ), the TS group was fed basal diet with transportation $(n=6)$, and the TS+GA group was fed basal diet+500 mg/kg of gallic acid $(\mathrm{GA})$ with transportation $(n=7)$.

protein) (51). The total dietary fiber (TDF) content was analyzed using an automatic fiber analyzer (FIBRETHERM FT12, C. Gerhardt GmbH \& Co. KG, Germany) and AOAC (2000; method 962.09 for crude fiber) (51). Diet was analyzed for GE by oxygen bomb calorimeter (IKA C 200, IKA (Guangzhou) Instrument Equipment Co., Ltd, Guangzhou, China).

\section{Fresh Fecal Sample Collection and Analysis}

During the whole experimental period of 2 weeks, fecal scores (FS) described by Middelbos et al. (52) were assessed every day. On BT1, AT1, and AT7, fresh fecal samples were collected from the pen floor of each dog within 15 min of defecation. An aliquot for SCFAs and branched-chain fatty acids (BCFAs) measurement was stored at $-80^{\circ} \mathrm{C}$ until analysis. An aliquot of the feces was collected and transferred to a 5 -ml sterile fecal collection tube (BIORISE) for microbiota measurement, snapfrozen on liquid $\mathrm{N}_{2}$, and stored at $-80^{\circ} \mathrm{C}$ until DNA extraction. Finally, an aliquot for metabolomics analysis was snap-frozen on liquid $\mathrm{N}_{2}$ and stored at $-80^{\circ} \mathrm{C}$ until analysis.

\section{Blood Sample Collection and Analysis}

On BT1, AT1, and AT7 after overnight fasting, a 5-ml blood sample was collected from each dog by forelimb vein and left to stand for $30 \mathrm{~min}$ before centrifugation at $3,500 \times \mathrm{g}$ at room temperature for $15 \mathrm{~min}$. After centrifugation, the supernatants were aliquoted into microcentrifuge tubes and stored at $-80^{\circ} \mathrm{C}$ for further analysis. Serum glutathione peroxidase (GSH-Px), malondialdehyde (MDA), total antioxidant capacity (T-AOC), and superoxide dismutase (SOD) were detected using commercial kits (Nanjing Jiancheng Bioengineering Institute, Nanjing, China) according to the manufacturer's protocol. Serum COR, GC, ACTH, HSP-70, immunoglobulin G (IgG), tumor necrosis factor-alpha (TNF- $\alpha$ ), interferon- $\gamma$ (IFN- $\gamma$ ), and interleukin 4 (IL-4) were measured using commercial ELISA kits (MEIMIAN, Jiangsu Meimian Industrial Co., Ltd., Jiangsu, China). Finally, an aliquot for serum metabolomics analysis was snap-frozen on liquid $\mathrm{N}_{2}$ and stored at $-80^{\circ} \mathrm{C}$ until analysis.

\section{S rRNA High-Throughput Sequencing DNA Extraction, Amplification, and Sequencing}

On BT1, AT1, and AT7, fresh fecal samples were collected from the pen floor of each dog within 15 min of defecation. Total genome DNA from fresh fecal samples was extracted using the cetyltrimethylammonium bromide method. DNA concentration and purity were monitored on $1 \%$ agarose gels. According to the concentration, DNA was diluted to $1 \mathrm{ng} / \mu \mathrm{l}$ using sterile water. $16 \mathrm{~S}$ rRNA genes of $16 \mathrm{~S} \mathrm{V3-V4} \mathrm{were} \mathrm{amplified} \mathrm{using} \mathrm{the} \mathrm{primers}$ 341F (5'-CCTAYGGGRBGCASCAG-3') and 806R (5' GGACTACNNGGGTATCTAAT- $3^{\prime}$ ) with the barcode. All PCRs were carried out with $15 \mu$ l of Phusion ${ }^{\circledR}$ High-Fidelity PCR Master Mix (New England Biolabs) with $2 \mu \mathrm{M}$ of forward and reverse primers and about $10 \mathrm{ng}$ of template DNA. Thermal cycling consisted of initial denaturation at $98^{\circ} \mathrm{C}$ for $1 \mathrm{~min}$, followed by 30 cycles of denaturation at $98^{\circ} \mathrm{C}$ for $10 \mathrm{~s}$, annealing at $50^{\circ} \mathrm{C}$ for $30 \mathrm{~s}$, and elongation at $72^{\circ} \mathrm{C}$ for $30 \mathrm{~s}$, followed by $72^{\circ} \mathrm{C}$ for $5 \mathrm{~min}$. The same volume of $1 \times$ loading buffer (contained SYB green) was mixed with PCR products (in equidensity ratios) and then operated with electrophoresis on $2 \%$ agarose gel for detection. Then, the mixture of PCR products was purified with Qiagen Gel Extraction Kit (Qiagen, Germany). Sequencing libraries were generated using the TruSeq ${ }^{\circledR}$ DNA PCR-Free Sample Preparation Kit (Illumina, USA) following the manufacturer's recommendations, and index codes were added. The library quality was assessed on the Qubit@ 2.0 Fluorometer (Thermo Scientific) and Agilent Bioanalyzer 2100 system. At last, 
the library was sequenced on an Illumina NovaSeq platform, and 250-bp paired-end reads were generated.

\section{Bioinformatics Analysis}

Paired-end reads were merged using FLASH (V1.2.7, http://ccb. jhu.edu/software/FLASH/) (53). Quality filtering on the raw tags was performed to obtain the high-quality clean tags (54) according to the QIIME (V1.9.1, http://qiime.org/scripts/split_ libraries_fastq.html) (55) quality-controlled process. The tags were compared with the reference database (Silva database, https://www.arb-silva.de/) using the UCHIME algorithm (UCHIME, http://www.drive5.com/usearch/manual/uchime_ algo.html) (56) to detect chimera sequences, and then the chimera sequences were removed (57). Then the effective tags are finally obtained.

Sequences analyses were performed by Uparse software (Uparse v7.0.1001, http://drive5.com/uparse/) (58). Sequences with $\geq 97 \%$ similarity were assigned to the same operational taxonomic units (OTUs). For each representative sequence, the Silva Database (http://www.arb-silva.de/) (59) was used based on the Mothur algorithm to annotate taxonomic information. Multiple sequence alignment was conducted using the MUSCLE software (Version 3.8.31, http://www.drive5.com/ muscle/) (60) to study the phylogenetic relationship of different OTUs. Alpha diversity indices, including Observed_species, Chao1, Shannon, Simpson, ACE, and PD_whole_tree, were calculated with QIIME (Version 1.7.0) and displayed with $\mathrm{R}$ software (Version 2.15.3). Beta diversity on weighted UniFrac was calculated by QIIME software (Version 1.9.1). Principal coordinate analysis (PCoA) based on weighted UniFrac distances was displayed by WGCNA package, stat packages, and ggplot2 package in $\mathrm{R}$ software (Version 2.15.3). The linear discriminant analysis (LDA) effect size (LEfSe) was processed with the default setting of LDA score $\geq 4$ using LEfSe software (http://huttenhower.sph.harvard.edu/lefse/). Correlation Network was performed using the OmicStudio tools at https://www.omicstudio.cn/tool. Function prediction of bacteria was conducted using PICRUSt (http://picrust.github. com/picrust/).

\section{Fecal Short-Chain Fatty Acid and Branched-Chain Fatty Acid Analyses}

\section{Sample Solution Preparation}

The fresh fecal samples collected on BT1, AT1, and AT7 were pretreated, and extraction of SCFAs and BCFAs was performed as follows. The frozen stool samples were placed on ice to thaw, and a 0.2 -g fecal sample was added with $1 \mathrm{ml}$ of ultra-pure water. After vortex for $2 \mathrm{~min}$, the samples were sonicated in an ice bath for $10 \mathrm{~min}$ and then centrifuged at $14,000 \mathrm{rpm}$ for $10 \mathrm{~min}$ at $4^{\circ} \mathrm{C}$. The supernatant was promptly transferred to a $2-\mathrm{ml}$ centrifuge tube, and then a total of $20 \mu \mathrm{l}$ of $25 \%$ metaphosphoric acid solution and $0.25 \mathrm{-g}$ anhydrous sodium sulfate were added to acidification and salting out, respectively. After vortex for $2 \mathrm{~min}$, $1 \mathrm{ml}$ of methyl tert-butyl ether was added, and the vortex was continued for $5 \mathrm{~min}$, and the supernatant was further centrifuged at $14,000 \mathrm{rpm}$ for another $10 \mathrm{~min}$ at $4^{\circ} \mathrm{C}$ to remove the precipitation. Finally, the upper extraction solution was harvested and filtered through $0.22-\mu \mathrm{m}$ Millipore pore membrane filters to a $2-\mathrm{ml}$ sample vial. Samples were stored at $-20^{\circ} \mathrm{C}$ until gas chromatography-MS (GC-MS) analysis. All steps above were performed at $4^{\circ} \mathrm{C}$ or on ice.

\section{Gas Chromatography-Mass Spectrometry Quantitative Analysis}

The quantitative analysis of SCFAs and BCFAs was carried out using the GCMS-QP2020 system (Shimadzu, Tokyo, Japan). The gas chromatography was equipped with an auto-injector AOC-20i (Shimadzu) and coupled to a flame ionization detector. The chromatographic separation was performed on a DB-FFAP capillary column $(30 \mathrm{~m} \times 0.25 \mathrm{~mm} \times 0.25 \mu \mathrm{m})$. Sample $(0.6 \mu \mathrm{l})$ was injected with a 30:1 split ratio using an autosampler. The injection port was set to a temperature of $250^{\circ} \mathrm{C}$. The initial temperature of the column was $80^{\circ} \mathrm{C}$ for $2 \mathrm{~min}$ and increased to $150^{\circ} \mathrm{C}$ at a rate of $10^{\circ} \mathrm{C} / \mathrm{min}$ for $2 \mathrm{in}$, and to $180^{\circ} \mathrm{C}$ at a rate of $15^{\circ} \mathrm{C} /$ $\min$ for $5 \mathrm{~min}$. The total run time was $18 \mathrm{~min}$. Helium (He; $99.999 \%$ ) was the carrier gas with a flow rate of $3 \mathrm{ml} / \mathrm{min}$. The MS parameters were electron impact mode at ionization energy of 70 $\mathrm{eV}$. The ion source and interface temperatures were $230^{\circ} \mathrm{C}$ and $250^{\circ} \mathrm{C}$, respectively. The solvent delay time was $1 \mathrm{~min}, 230^{\circ} \mathrm{C}$. The acquisition mode was selected at ion monitoring mode with a scan interval of $0.3 \mathrm{~s}$.

\section{Fecal and Serum Untargeted Metabolomics Analyses}

\section{Sample Processing}

The fresh fecal and serum samples collected on BT1, AT1, and AT7 were processed as described previously (61) with slight modifications. Briefly, frozen stool samples stored at $-80^{\circ} \mathrm{C}$ were thawed at $4^{\circ} \mathrm{C}$. Approximately $60 \mathrm{mg}$ of sample was weighed and put into $2-\mathrm{ml}$ round-bottom microcentrifuge tubes. Metabolites were extracted by adding $600 \mu \mathrm{l}$ of methanol:water $(1: 1, \mathrm{v} / \mathrm{v})$, and magnetic beads were added to the microcentrifuge tubes for homogenization using a homogenizer. Ultrasonic crushing was performed at a low temperature for $10 \mathrm{~min}$, followed by $-20^{\circ} \mathrm{C}$ for $30 \mathrm{~min}$. The samples were then centrifuged at $14,500 \mathrm{rpm}$, $4^{\circ} \mathrm{C}$ for $15 \mathrm{~min}$, and $200 \mu \mathrm{l}$ of supernatant was dried in a vacuum centrifuge. Immediately afterward, the samples were redissolved with $200 \mu \mathrm{l}$ of $50 \%$ methanol each and vortexed for $2 \mathrm{~min}$. After ultrasonic crushing for $10 \mathrm{~min}$ at a low temperature, the microcentrifuge tube was centrifuged again at $14,500 \mathrm{rpm}, 4^{\circ} \mathrm{C}$ for $15 \mathrm{~min}$. Finally, the supernatant was stored in a sample injection bottle for analysis. Meanwhile, to prepare for the quality control (QC) sample, $100 \mu \mathrm{l}$ of supernatant from each sample was taken in a $15-\mathrm{ml}$ centrifuge tube in order to examine the stability and reproducibility of the entire analysis process. Frozen serum samples collected on BT1, AT1, and AT7 were thawed at $4^{\circ} \mathrm{C}$, and vortexed for $2 \mathrm{~min}$. For each sample, $200 \mu \mathrm{l}$ of serum sample, $800 \mu \mathrm{l}$ of methanol, and $10 \mu \mathrm{l}$ of indole acetic acid ethyl ester (internal standard) were sequentially added to the 1.5$\mathrm{ml}$ RNAase-free centrifuge tube and vortexed for $2 \mathrm{~min}$. The samples were then centrifuged at $14,500 \mathrm{rpm}, 4^{\circ} \mathrm{C}$ for $15 \mathrm{~min}$; and $800 \mu \mathrm{l}$ of supernatant was dried in a vacuum centrifuge for 
$3 \mathrm{~h}$, blow-dried with nitrogen, and processed immediately. The next operation processes and QC sample preparation were similar to those of fecal samples.

\section{Multivariate Analysis}

UPLC-Orbitrap-MS/MS analysis method was carried out as described previously (62), with minor modifications. The Compound Discoverer 2.1 (Thermo Fisher Scientific) data analysis tool was employed to automate complete raw data preprocessing and was applied to identify metabolites by searching the mzCloud library and mzVault library. In this study, MetaboAnalyst 5.0 (https://www.metaboanalyst.ca) was used to perform multivariate analysis. Principal component analysis (PCA) and orthogonal partial least-squares discriminant analysis (OPLS-DA) of metabolites were performed. Pathway enrichment analysis was performed by using the enrichment analysis module on MetaboAnalyst 5.0. The visualization results of the models were obtained with MetaboAnalyst 5.0.

\section{Statistical Analysis}

SPSS 26.0 and GraphPad Prism 8.0 software were used for statistical analysis and graphical presentation. One-way ANOVA followed by the multiple range test of least significant difference was used to determine the statistical significance of multiple comparisons. All data were expressed as the mean \pm standard error (SE). Significant differences were at $p<0.05$, and tendencies were at $p<0.10$. To preliminarily screen the differential metabolites, we selected the metabolites that had a $p$-value of less than 0.05 (calculated by Student's t-test) and a variable importance in projection (VIP) score greater than 1.0 (calculated using Orthogonal PLS-DA model). Spearman's correlation values and significance were computed with the $\mathrm{R}$ version 3.6.1. Clustering correlation heatmap with signs was performed using the OmicStudio tools at https://www. omicstudio.cn.

\section{RESULTS}

\section{Effect of Gallic Acid on Fecal Scores, Serum Hormone, HSP-70, Antioxidant Capacity, and Inflammatory Factors in Puppies}

Changes in FS are shown in Figure 2A. It is evident that the TS+GA group had lower FS than the CON or TS group on BT6 $(p<0.01)$, BT3 $(p<0.05)$, and BT1 $(p=0.085)$. And we found that FS increased in the TS group on AT1 $(p=0.085)$ and AT2 $(p<0.05)$. During the whole experimental period (Figure 2B), puppies fed GA $(2.61 \pm 0.05)$ had a normal fecal shape relative to the CON $(3.17 \pm$ $0.07)$ and TS groups $(3.13 \pm 0.07)(p<0.001)$. Total diarrhea rate (TDR) in the CON, TS, and TS+GA groups were $26.5 \%, 22.6 \%$, and $4.1 \%$, respectively, and GA reduced TDR by as much as $84.5 \%$ and $81.9 \%$ compared with the CON and TS groups, respectively.

Puppies fed a diet containing $500 \mathrm{mg} / \mathrm{kg}$ of GA for 7 days had a trend toward lower COR compared with the CON group $(p=0.06$,
Figure 2C). Over time, there was no significant change. The TS group displayed a higher glucocorticoid (GC) level on AT7 $(p<$ 0.01; Figure 2D). Similarly, ACTH acts on the adrenal cortex and stimulates GC and COR secretion; thus, it had a similar change as GC and COR (Figure 2E). No difference in HSP-70 was observed on BT1 and AT1 (Figure 2F); however, over time, the TS group had a higher HSP-70 level than the CON group on AT7 $(p<0.05)$, and puppies fed GA had no significant change as compared with the TS group.

There was no different GSH-Px activity between the CON and TS groups on BT1 and AT1 (Figure 2G), while puppies fed GA had higher GSH-Px activity than the CON group on AT1. And a decreasing trend of GSH-Px activity was observed in the TS group over the CON group on AT7 ( $p=0.057)$. Dietary GA supplementation markedly improved the GSH-Px activity after transportation $(p<0.01)$. Additionally, the TS group had a marginally higher MDA level than the CON group on AT1 ( $p=$ 0.058 , Figure $\mathbf{2 H}$ ), whereas the TS group had a decreasing trend of MDA than the CON group ( $p=0.061)$, and the TS+GA group had a decreasing MDA level over the CON group on AT7 $(p<0.05)$. The T-AOC and SOD contents had no obvious change among groups (Figures 2I, J).

The TS group tended to decrease the serum IgG level on AT1 relative to the CON group ( $p=0.093$, Figure $2 \mathbf{K}$ ), while puppies fed GA had a higher IgG level than the TS group $(p<0.05)$. Though both the CON and TS+GA groups had surprisingly higher TNF- $\alpha$ levels than the TS group on BT1 $(p=0.059, p<$ 0.05 , Figure 2L), the TS group had a higher TNF- $\alpha$ level than the CON group over time on AT7 $(p<0.05)$, and no significant difference was observed between the TS+GA and TS groups. Similarly, the TS and TS+GA groups showed an unexpected increase in IFN- $\gamma$ level over the CON group on BT1 $(p=0.053$, $p<0.05$, Figure $2 \mathbf{M}$ ), but there was no difference among groups after transportation. Furthermore, IL-4 level sharply decreased in the TS group over the CON group on AT7 $(p<0.01$, Figure $2 \mathrm{~N}$ ), while puppies fed GA had a significant increase of IL-4 level than the TS group $(p<0.01)$.

\section{Effect of Gallic Acid on Gut Microbial Composition and Structure in Puppies}

On BT1, puppies fed a basal diet at $500 \mathrm{mg} / \mathrm{kg}$ of GA for 7 days had more Observed_species and higher Chaol and ACE indices than those of the CON group ( $p<0.05$, Figure 3A). No difference was observed among the three groups on AT1 and AT7. From the difference of beta diversity index based on weighted UniFrac distances, PCoA plots revealed distinct separation between the CON and TS+GA groups on BT1 and AT1 $(p<0.05$, Figure 3B), whereas the CON group had a trend toward significant separation relative to the TS group on AT7 ( $p=$ $0.095)$, especially that puppies fed the dietary supplementation of GA had distinct separation over the TS group $(p<0.05)$.

The most abundant phyla included Firmicutes (60.24\%), Actinobacteria (11.47\%), Fusobacterota (6.52\%), Actinobacteriota (3.52\%), Proteobacteria (3.24\%), and Bacteroidota (1.13\%) at various time points (Figure 3C). Puppies fed GA had the highest Firmicutes abundance on AT1 and tended to have higher 
A
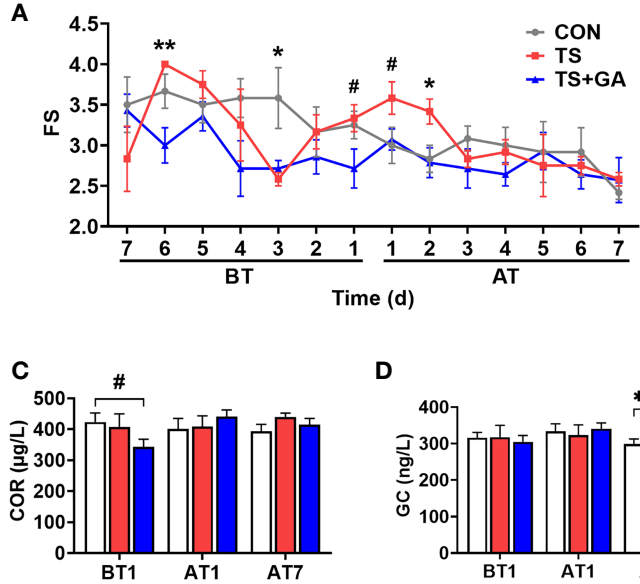

D

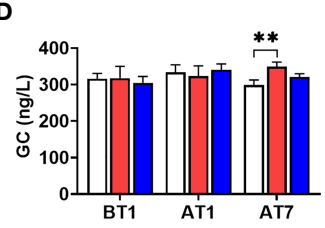

H

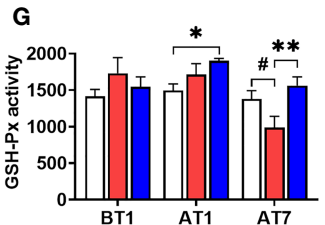

K

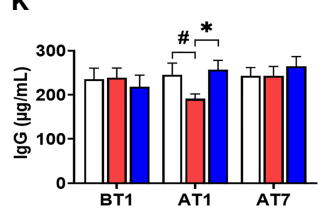

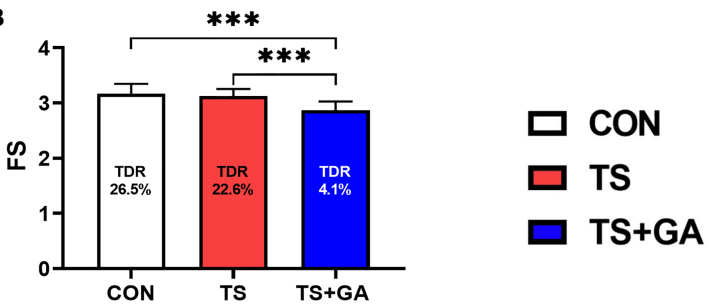

E
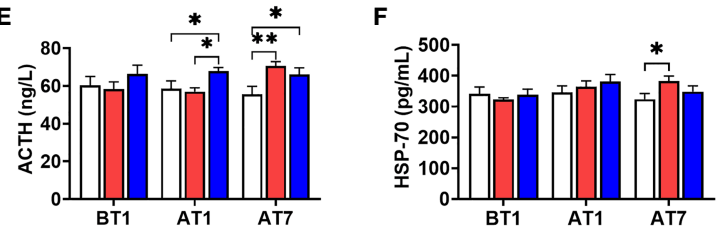

J
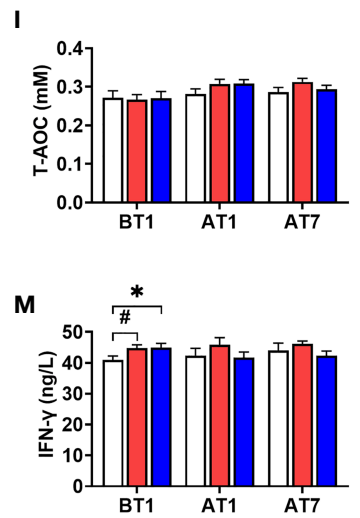

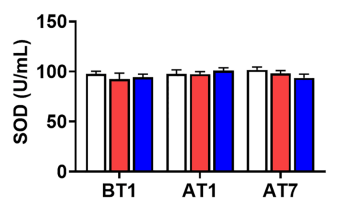

N

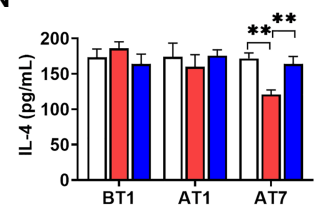

FIGURE 2 | Effect of gallic acid (GA) on fecal score (FS) (A, B), serum hormone (C-E), HSP-70 (F), antioxidant capacity (G-J), and inflammatory factors (K-N) in puppies ( $\mathrm{n}=6$ or 7 ). The symbol $\left({ }^{\star}\right)$ indicates statistically significant differences between two groups $\left({ }^{\star} p<0.05,{ }^{\star \star} p<0.01\right.$, and $\left.{ }^{\star \star \star} p<0.001\right)$, and the symbol $(\#)$ represents difference tendency (\#p <0.10). BT1, the 1st day before transportation; AT1, the 1st day after transportation; AT7, the 7th day after transportation. TDR (total diarrhea rate, \%) = [cases of diarrhea during 14 days/(14 days $\times$ total puppies for each group)] $\times 100$. COR, cortisol; ACTH, adrenocorticotropic hormone; GC, glucocorticoid; HSP-70, heat stress protein 70; GSH-Px, glutathione peroxidase; MDA, malondialdehyde; T-AOC, total antioxidant capacity; SOD, superoxide dismutase; IgG, immunoglobulin G; TNF- $\alpha$, tumor necrosis factor- $\alpha$; IFN- $\gamma$, interferon- $\gamma$; IL-4, interleukin 4.

Firmicutes than the CON group $(p=0.055)$. Furthermore, GA caused inhibition of Proteobacteria growth induced by transportation stress $(p<0.05)$. Also, the most abundant families included Erysipelotrichaceae (23.28\%), Peptostreptococcaceae (12.53\%), Lachnospiraceae (12.22\%), Bifidobacteriaceae (11.36\%), Peptostreptococcaceae (7.18\%), Lactobacillaceae (6.60\%), and Fusobacteriaceae $(6.52 \%)$ at various phases. Decreasing Peptostreptococcaceae abundance was observed in the TS+GA group compared with the CON group on AT1 $(p<0.05)$. In contrast, a relative abundance of Lactobacillaceae was higher in the TS+GA group compared with the CON group on AT1 $(p<0.05)$. Relative abundance of Eggerthellaceae in the TS group significantly increased over the CON group, and the TS group had a higher Eubacteriaceae abundance than the CON and TS+GA groups $(p<$ $0.05)$. Finally, the most abundant genera were Allobaculum (16.08\%), Bifidobacterium (11.36\%), Peptoclostridium (11.08\%), Blautia (8.48\%), Lactobacillus (6.60\%), Turicibacter (5.26\%), Cetobacterium (4.40\%), Escherichia-Shigella (2.46\%), Streptococcus
(2.33\%), Fusobacterium (2.08\%), Collinsella (1.67\%), and Faecalibacterium (1.27\%). Relative abundance of Romboutsia significantly decreased in the TS+GA group compared with the CON group on BT1. Relative abundances of Lactobacillus and Faecalibaculum were higher, and relative abundances of Escherichia-Shigella and Clostridium_sensu_stricto_1 were lower in the TS+GA group compared with the CON or TS group on AT1 $(p<0.05)$. The TS group had a higher relative abundance of Allobaculum and Dubosiella than the CON group on AT7 $(p<$ 0.05 ), while no difference was observed in the TS+GA group; and both the TS and TS+GA groups had lower Turicibacter relative to the CON group $(p<0.05)$.

Differential taxon abundances were further confirmed by LEfSe analysis. The histogram with logarithmic LDA score $>4.0$ and cladogram is shown in Figure 4A. On BT1, the LEfSe analysis indicated that Peptostreptococcaceae and Streptococcus in the CON group were the most abundant, whereas on AT1, the predominant bacterial strains in the TS group were Escherichia- 


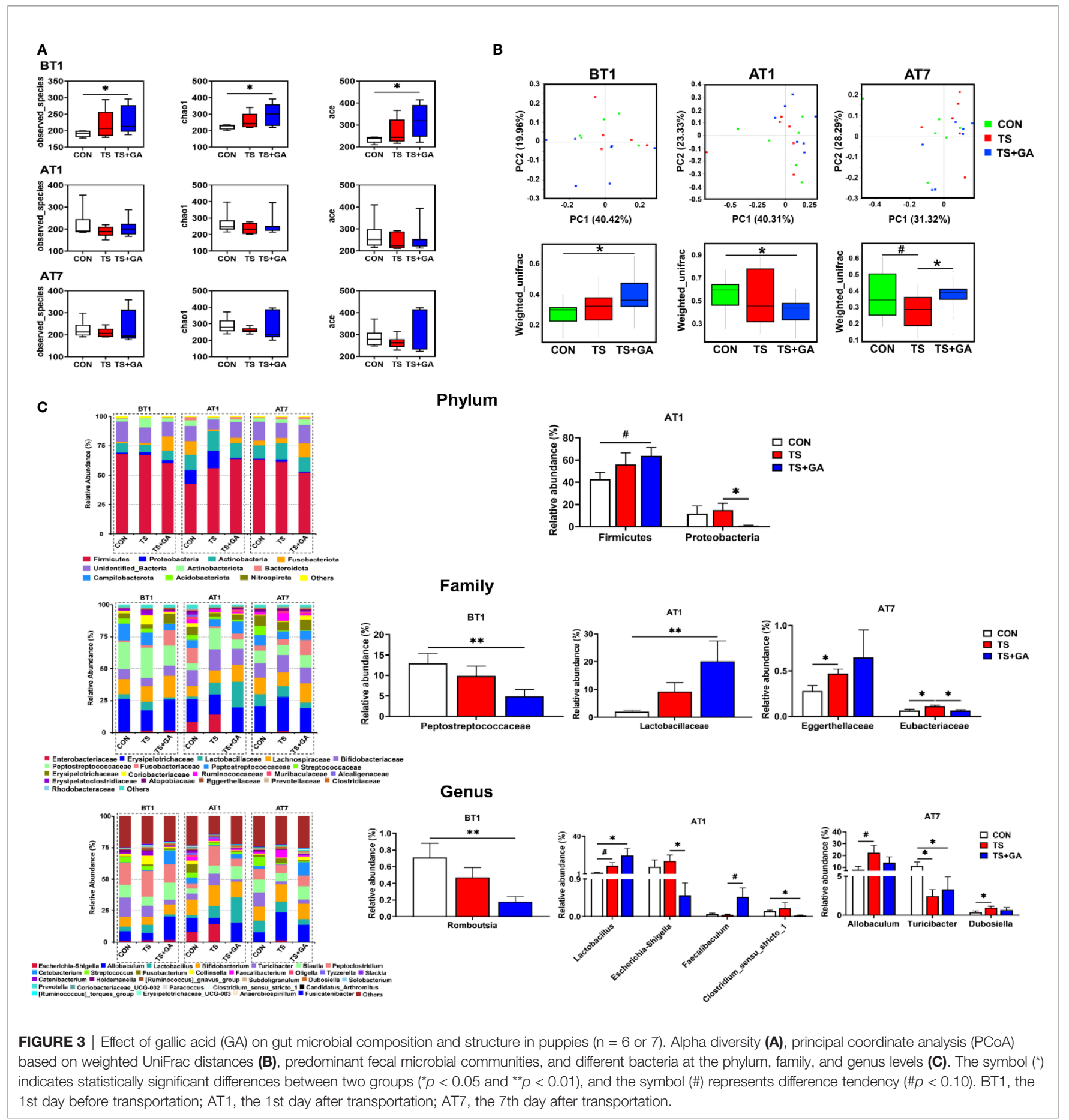

Shigella and Escherichia coli. Fortunately, Lactobacillus, Lactobacillus murinus, and Lactobacillus reuteri were the highest in the TS+GA group, while no difference was observed on AT7. We next determined the relationship and interaction among fecal microbiota using Spearman's correlation analysis. As shown in Figure 4B, Escherichia-Shigella negatively modulated Faecalibaculum, Lactobacillus, and Bifidobacterium and positively modulated Streptococcus and Clostridium_ sensu_stricto_1 in the network. Allobaculum positively modulated Faecalibaculum, Dubosiella, Cetobacterium, and Fusobacterium. There was a positive network among Catenibacterium, Prevotella, Collinsella, [Ruminococcus]_gnavus_group, Holdemanella, Blautia, and Peptoclostridium. In addition, we also found a positive correlation between Romboutsia and Turicibacter. Regarding Spearman's analysis, the whole network of microbiota was divided into several parts, in which genera Escherichia-Shigella, 

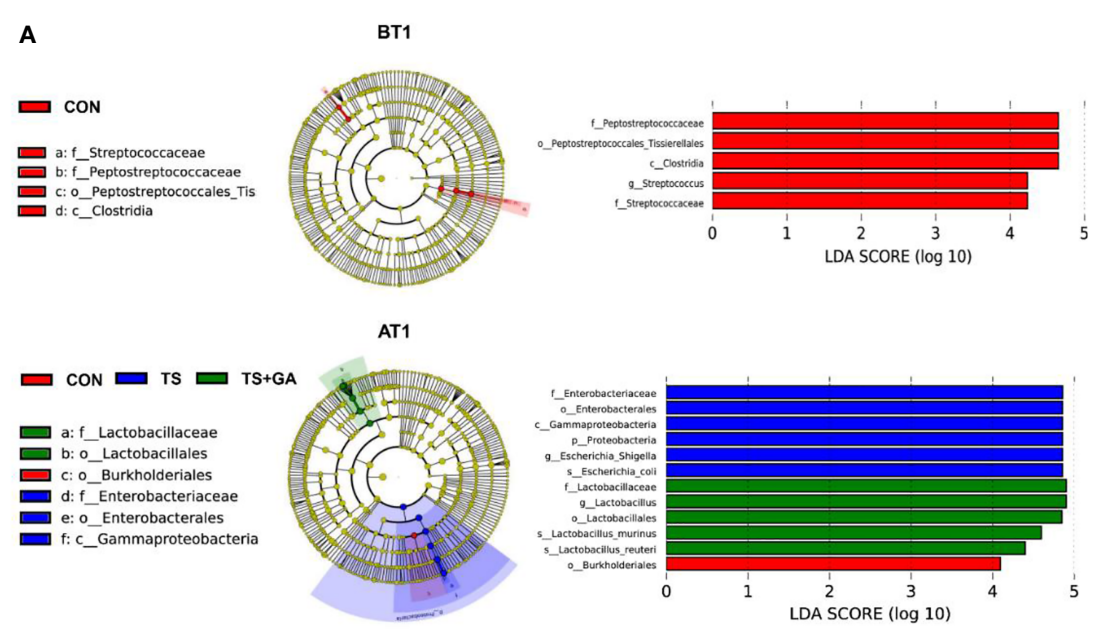

B

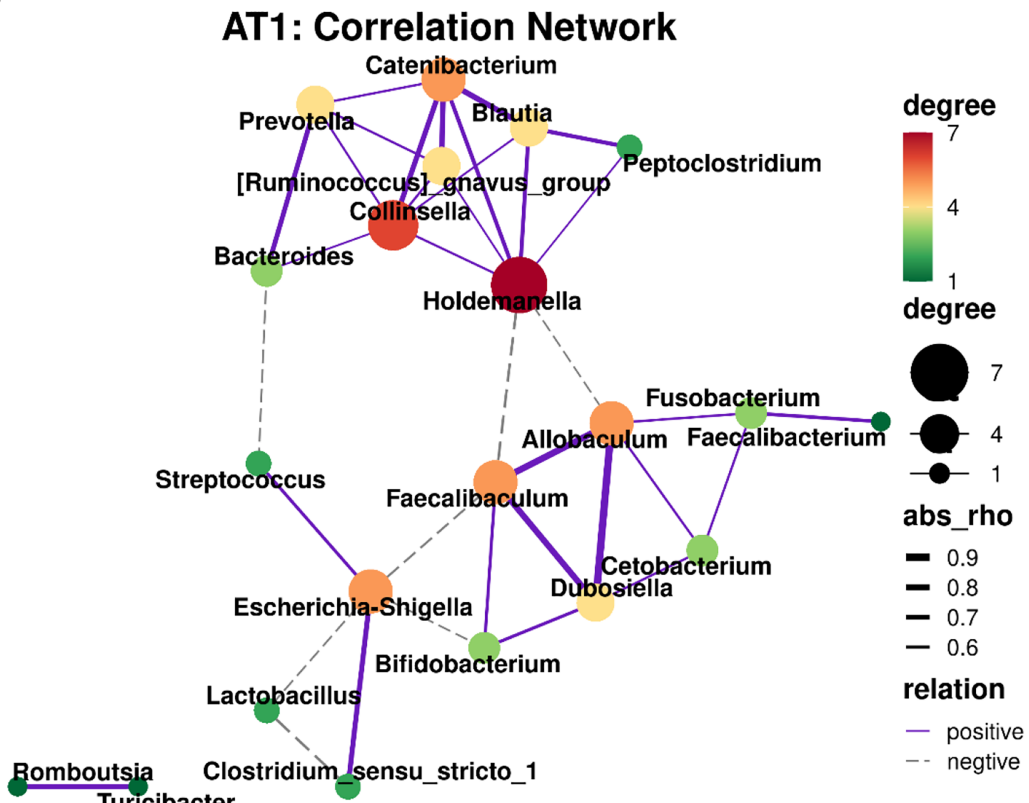

FIGURE 4 | The linear discriminant analysis effect size (LEfSe) analysis identified gut bacterial biomarkers in puppies on BT1 and AT1 (A). Spearman's correlation network of fecal microbiota at genus level on AT1 (purple solid line, positive correlation; gray dotted line, negative correlation; thick line, significant correlation, $p<$ 0.05) (B). BT1, the 1st day before transportation; AT1, the 1st day after transportation.

Allobaculum, Catenibacterium, and Holdemanella dominated key positions and had close interactions with many bacteria in the community.

The gut bacterial function and pathways after transportation and GA treatment were predicted by PICRUSt analysis based on the Kyoto Encyclopedia of Genes and Genomes (KEGG) pathway using $16 \mathrm{~S}$ rRNA data. On BT1, puppies fed GA had more abundant amino acid metabolism, energy metabolism, carbohydrate metabolism, nucleotide metabolism, metabolism of cofactors and vitamins, and metabolism of terpenoids and polyketide (Figure S1A), indicating that these metabolic pathways were significantly influenced by GA in the short term. It is worth noting that the decreasing abundance of genes involved in energy metabolism and glycan biosynthesis and metabolism were found in the TS+GA group relative to the CON group on AT1 (Figure S1B), while more abundant carbohydrate metabolism was observed in the TS+GA group over the TS group. On AT7, puppies transported to another livable environment had weaker amino acid metabolism and xenobiotics biodegradation and metabolism than the CON group (Figure S1C), whereas energy metabolism, xenobiotics biodegradation and metabolism, and metabolism of cofactors and vitamins were markedly enhanced after GA treatment compared with those of the CON group. 


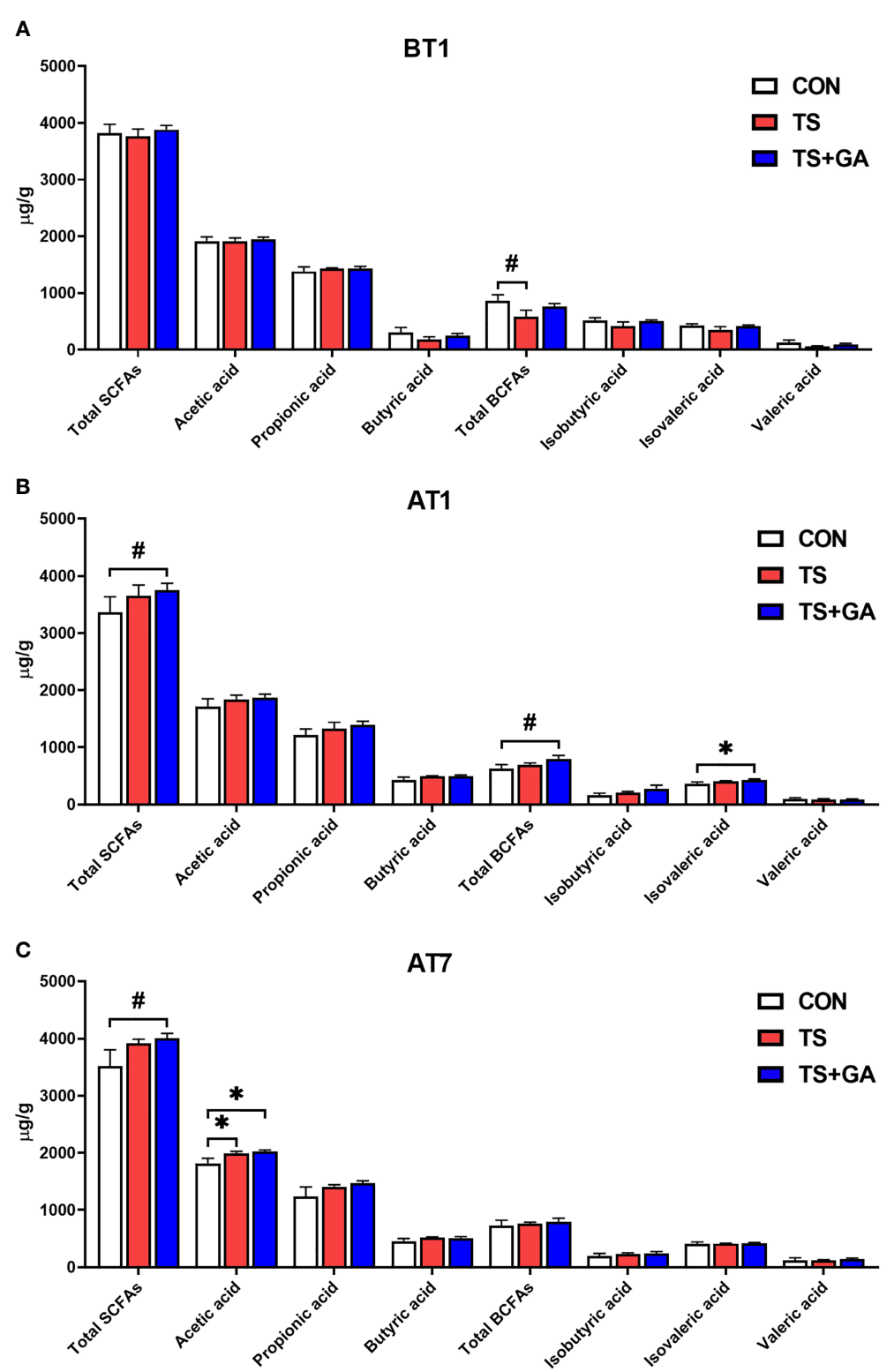

FIGURE 5 | Effect of gallic acid (GA) on fecal short-chain fatty acids (SCFAs) and branched-chain fatty acids (BCFAs) in puppies on BT1 (A), AT1 (B), and AT7 (C) $(\mathrm{n}=6$ or 7$)$. The symbol $\left(^{*}\right)$ indicates statistically significant differences between two groups $\left({ }^{*} p<0.05\right)$, and the symbol (\#) represents difference tendency $(\# p<$ 0.10). BT1, the 1st day before transportation; AT1, the 1st day after transportation; AT7, the 7th day after transportation.

\section{Effect of Gallic Acid on Fecal Short-Chain Fatty Acids and Branched-Chain Fatty Acids in Puppies}

No significant differences in SCFAs concentrations were observed among the three groups except for total BCFAs between the CON and TS groups on BT1 $(p=0.057$; Figure 5A), while puppies fed GA had a trend of increase in total SCFAs ( $p=0.083$; Figure 5B) and increasing total BCFAs $(p=0.087)$ and isovaleric acid $(p<0.05)$ content relative to the CON group on AT1. Similarly, the TS+GA group had a similar trend of increase in total SCFAs to the CON group $(p=0.099$;
Figure 5C), and higher acetic acid levels were observed in the TS and TS+GA groups in comparison with the CON group on AT7 $(p<0.05)$.

\section{Effect of Gallic Acid on Fecal Metabolites in Puppies}

Multivariate statistical analysis was carried out among three groups. In this study, the PCA was used to study the differences among the CON, TS, and TS+GA groups in the fecal metabolomics by an unsupervised statistical method (Figure 6A). The PCA score plots showed less obvious 

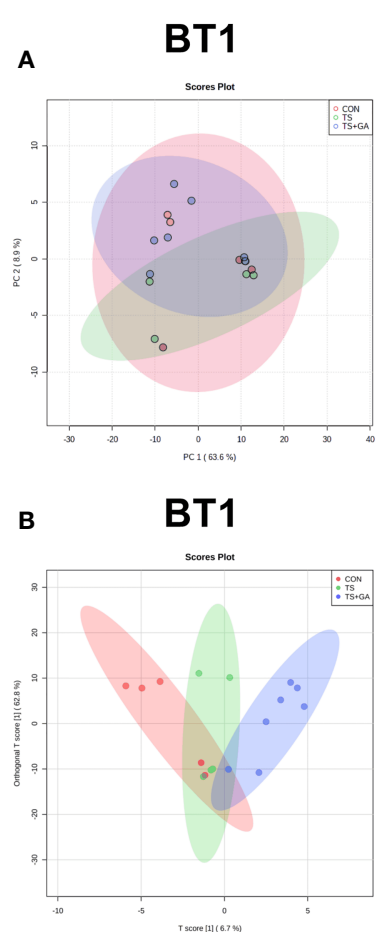

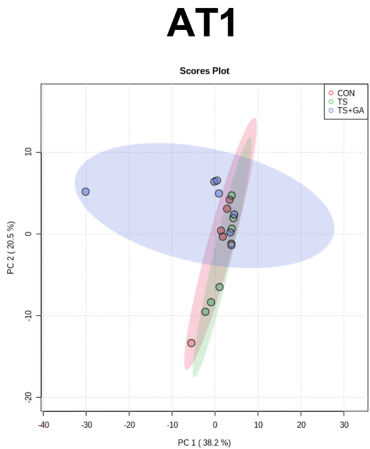

AT1

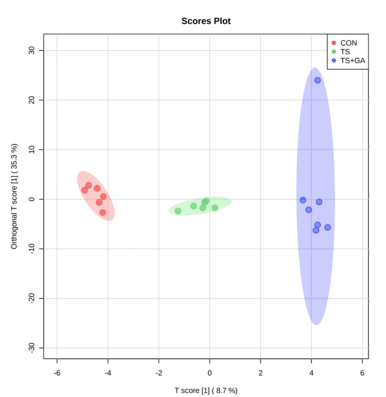

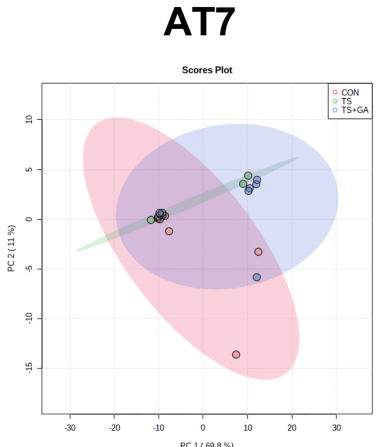

AT7

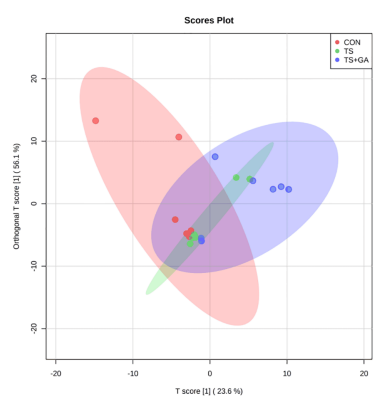

FIGURE 6 | Multivariate statistical analysis on BT1, AT1, and AT7 ( $\mathrm{n}=6$ or 7). Score plots from the principal component analysis (PCA) model among three groups on BT1, AT1, and AT7 (A). Score plots from the orthogonal partial least-squares discriminant analysis (OPLS-DA) model among three groups on BT1, AT1, and AT7 (B). BT1, the 1st day before transportation; AT1, the 1st day after transportation; AT7, the 7th day after transportation.

separation at varying time points. However, the OPLS-DA model revealed a clearer difference between the three clusters on AT1 (Figure 6B), indicating that the difference among the three groups was the most obvious when puppies were transported from a stressful environment to another livable location.

In this study, a total of 156 metabolites were detected at all stages (Table S2). The differential metabolites at varying time points are shown in Table S3. A total of 6, 16, and 8 potential biomarkers were identified on BT1, AT1, and AT7, respectively. To gain further insight into the metabolic changes, a KEGG pathway analysis of all metabolites was performed. On BT1, the influenced pathway was mainly concentrated in glycan biosynthesis and metabolism (glycosylphosphatidylinositol (GPI)-anchor biosynthesis) (Figure 7A). On AT1, the most influenced metabolic pathways were amino acid metabolism (phenylalanine metabolism, tyrosine metabolism; phenylalanine, tyrosine, and tryptophan biosynthesis; valine, leucine, and isoleucine degradation; and valine, leucine, and isoleucine biosynthesis), lipid metabolism (steroid hormone biosynthesis and glycerolipid metabolism), metabolism of cofactors and vitamins (ubiquinone and other terpenoid-quinone biosynthesis, and pantothenate and CoA biosynthesis), and carbohydrate metabolism (fructose and mannose metabolism) (Figure 7B). On AT7, the most important metabolic pathways were carbohydrate metabolism (purine metabolism, and glyoxylate and dicarboxylate metabolism), amino acid metabolism (tryptophan metabolism), and glycan biosynthesis and metabolism (GPI-anchor biosynthesis) (Figure 7C). As a result, we found that the significant differences in the metabolic pathways were mainly concentrated in AT1. The levels of predominant potential biomarkers based on the significant metabolic pathways on BT1, AT1, and AT7 are shown in Table S4.

\section{Effect of Gallic Acid on Serum Metabolites in Puppies}

Based on the fecal metabolomics analysis, we further detected serum metabolomics. As shown in Figure 8A, the PCA score plots showed distinct separation among the CON, TS, and TS +GA groups after transportation. Similarly, the score plots for the OPLS-DA model presented clear separation over time (Figure 8B), suggesting a difference among the three groups. From these results of multivariate statistical analysis, it is apparent that there are greater differences in serum metabolites than fecal metabolites at different stages.

In this study, a total of 147 metabolites were detected at all stages (Table S5). The differential metabolites at varying time points are shown in Table S6. A total of 13, 48, and 36 potential biomarkers were identified on BT1, AT1, and AT7, respectively. On BT1, puppies fed GA mainly influenced serum amino acid metabolism (lysine degradation, tyrosine metabolism, taurine and hypotaurine metabolism, and glutathione metabolism), carbohydrate metabolism (glycolysis/gluconeogenesis and pyruvate metabolism), and lipid metabolism (sphingolipid 


\section{BT1: CON vs. TS}

A

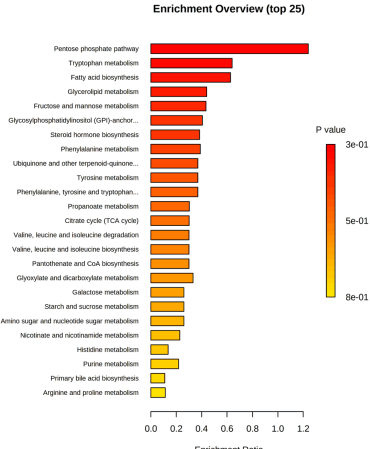

AT1: CON vs. TS

B

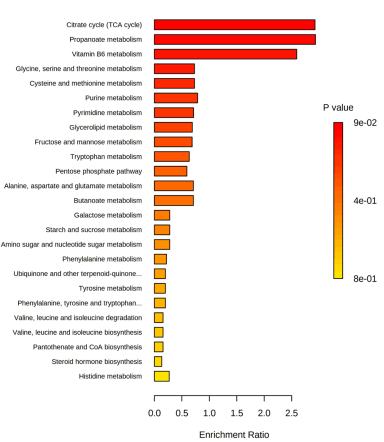

AT7: CON vs. TS

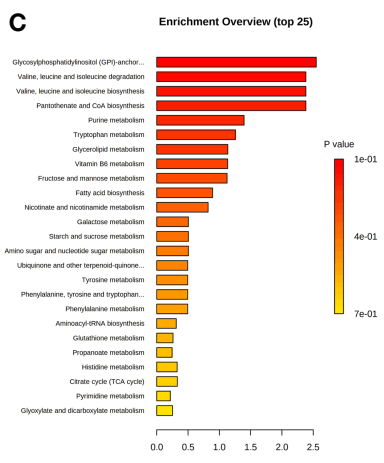

BT1: CON vs. TS+GA

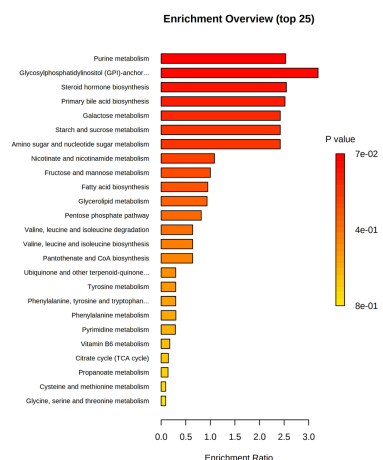

AT1: CON vs. TS+GA

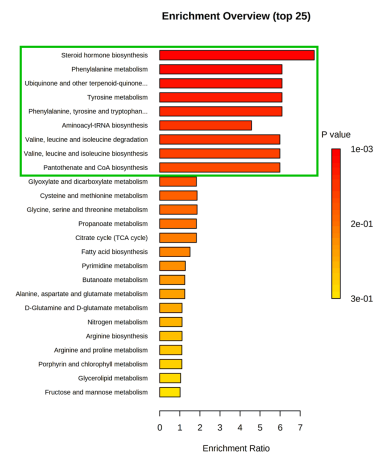

AT7: CON vs. TS+GA

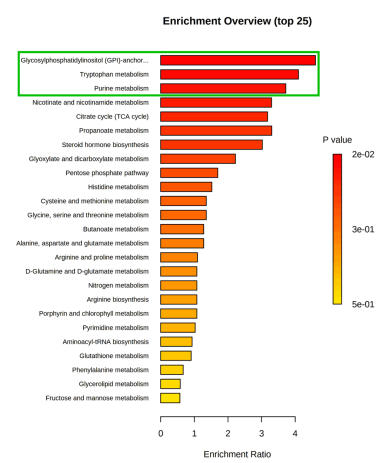

BT1: TS vs. TS+GA

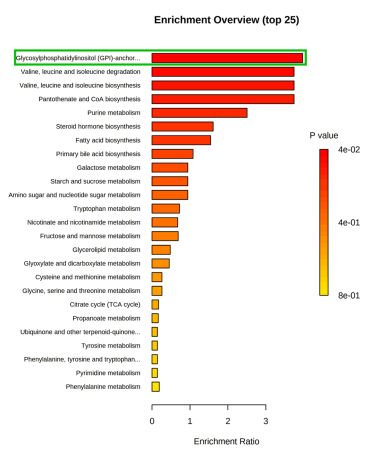

AT1: TS vs. TS+GA

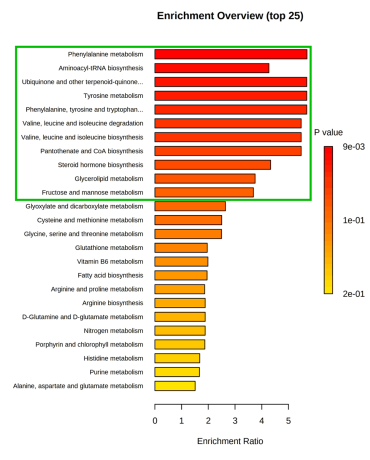

AT7: TS vs. TS+GA

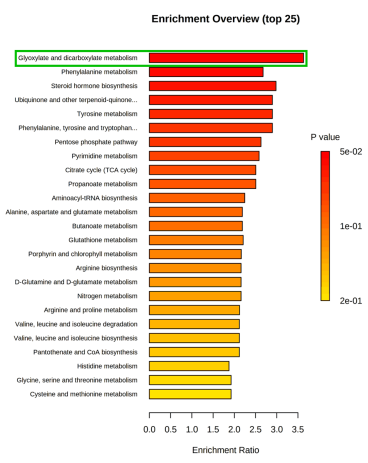

FIGURE 7 | Bar charts of the metabolic pathway analysis of differential fecal metabolites on BT1 (A), AT1 (B), and AT7 (C) ( $\mathrm{n}=6$ or 7$)$. The pathway enrichment analysis shows all matched pathways, and the green boxes indicate significant metabolic pathways $(p<0.05)$. BT1, the 1st day before transportation; AT1, the 1st day after transportation; AT7, the 7th day after transportation.

metabolism) (Figure 9A). On AT1, the influenced pathway was mainly concentrated in amino acid metabolism (glycine, serine, and threonine metabolism; arginine and proline metabolism; arginine biosynthesis, alanine, aspartate, and glutamate metabolism; and D-glutamine and D-glutamate metabolism), carbohydrate metabolism (glyoxylate and dicarboxylate metabolism), energy metabolism (nitrogen metabolism), and nucleotide metabolism (pyrimidine metabolism) between the CON and TS groups (Figure 9B), whereas feeding GA was implicated in the regulation of carbohydrate metabolism (glycolysis/gluconeogenesis and pyruvate metabolism) and lipid metabolism (alpha-linolenic acid metabolism, linoleic acid metabolism, and biosynthesis of unsaturated fatty acids) compared with the other two groups. On AT7, the affected 
pathways mainly involved amino acid metabolism (tyrosine metabolism and cysteine and methionine metabolism) and lipid metabolism (sphingolipid metabolism and fatty acid biosynthesis) in the TS group compared with the CON group (Figure 9C); notably, significant enrichment of several major metabolic pathways, such as amino acid metabolism (cysteine and methionine metabolism; tyrosine metabolism; valine, leucine, and isoleucine degradation; valine, leucine, and isoleucine biosynthesis; lysine degradation; and taurine and hypotaurine metabolism), carbohydrate metabolism (glycolysis/ gluconeogenesis, pyruvate metabolism, and fructose and mannose metabolism), lipid metabolism (glycerolipid metabolism, fatty acid biosynthesis, primary bile acid biosynthesis, and alpha-linolenic acid metabolism), and nucleotide metabolism (purine metabolism), was significantly changed by GA. The levels of predominant potential biomarkers based on the significant metabolic pathways on BT1, AT1, and AT7 were presented in Table S7.

\section{The Correlation Analysis of Metabolites and Microbiota}

Spearman's correlation analysis was performed for the differential feces and serum metabolites and fecal microbiota obtained by high-throughput $16 \mathrm{~S}$ rRNA sequencing. On AT1, we found that fecal L-arginine, L-valine, phenylacetaldehyde, and tetrahydrodeoxycorticosterone were positively correlated with the relative abundance of Clostridium_sensu_stricto_1 (Figure 10A). And glyceraldehyde, L-glutamic acid, L-tyrosine, L- valine, and tetrahydrodeoxycorticosterone were positively correlated with the relative abundance of Escherichia-Shigella. LGlutamic acid, L-tyrosine, and L-valine were also positively correlated with Proteobacteria. Conversely, L-tyrosine and phenylacetaldehyde were negatively correlated with Lactobacillaceae and Lactobacillus. In addition, we also observed a weak positive association between Faecalibaculum with total BCFAs (isobutyric acid and isovaleric acid), and the total SCFAs (acetic acid and propionic acid) had a weak positive association with Firmicutes. On AT7, uridine diphosphate-Nacetylglucosamine was negatively correlated with the relative abundance of Turicibacter. Furthermore, butyric acid and isobutyric acid had a weak positive association with the allobaculum.

As shown in Figure 10B, alpha-linolenic acid, citric acid, Llactic acid, oleic acid, and spermidine were positively correlated with Clostridium_sensu_stricto_1 on AT1. Likewise, alphalinolenic acid, L-lactic acid, and oleic acid were also positively correlated with Escherichia-Shigella. And a significant positive association was found between L-lactic acid with Proteobacteria. In contrast, alpha-linolenic acid, oleic acid, and spermidine were negatively correlated with Lactobacillaceae and Lactobacillus. Additionally, strong negative and positive associations of the Larginine with Faecalibaculum and the arachidonic acid with Firmicutes were observed. On AT7, serum phytosphingosine and taurochenodeoxycholic acid had a reverse association with Eggerthellaceae. Similarly, glyceraldehyde and L-carnitine had positive and negative associations with Turicibacter. And
A BT1

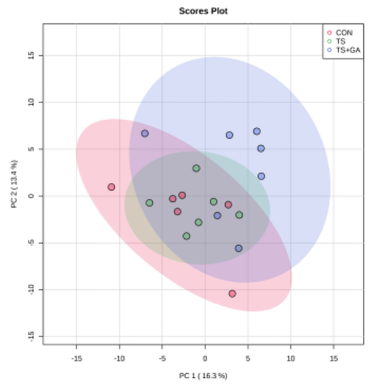

B

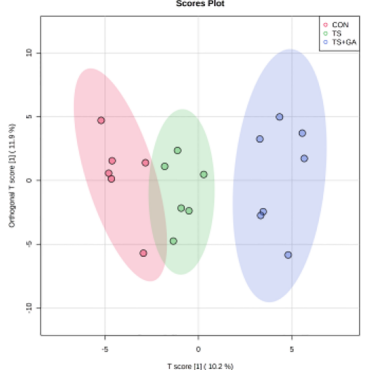

AT1

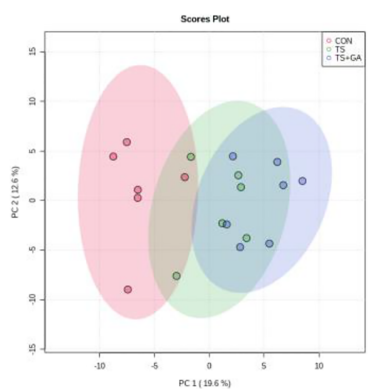

AT1

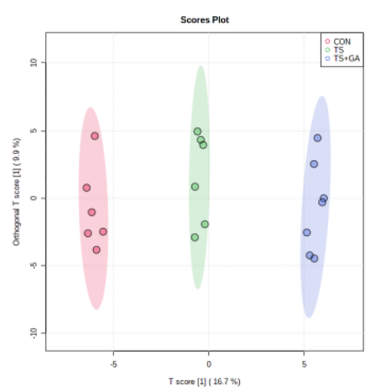

AT7

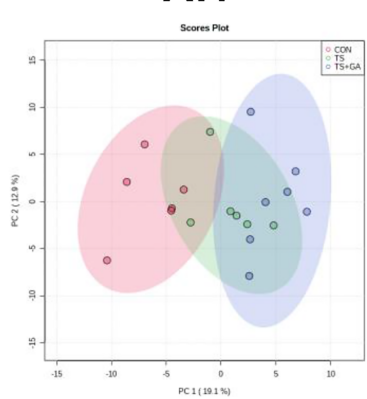

AT7

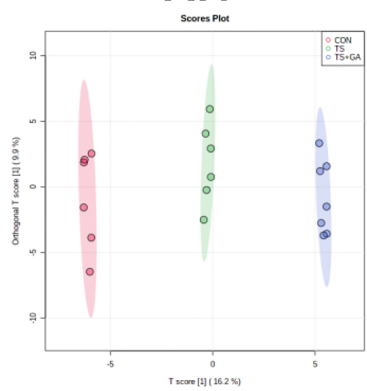

FIGURE 8 | Multivariate statistical analysis on BT1, AT1, and AT7 ( $n=6$ or 7). Score plots from the principal component analysis (PCA) model among three groups on BT1, AT1, and AT7 (A). Score plots from the orthogonal partial least-squares discriminant analysis (OPLS-DA) model among three groups on BT1, AT1, and AT7 (B). BT1, the 1st day before transportation; AT1, the 1st day after transportation; AT7, the 7th day after transportation. 


\section{BT1: CON vs. TS}

A

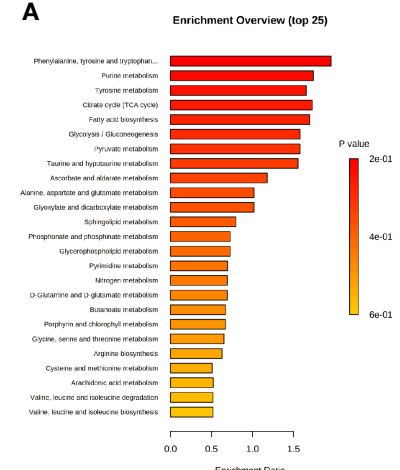

AT1: CON vs. TS

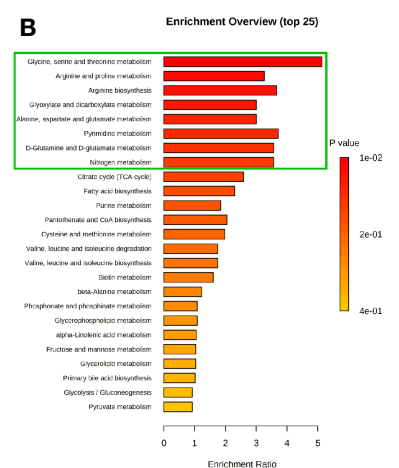

AT7: CON vs. TS

C

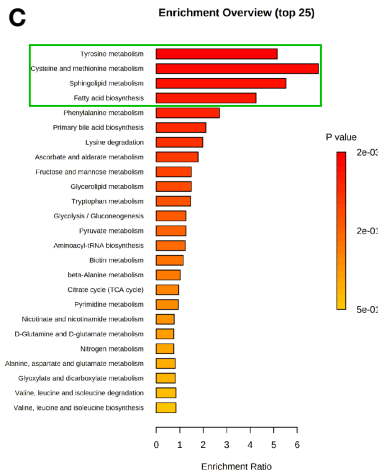

BT1: CON vs. TS+GA

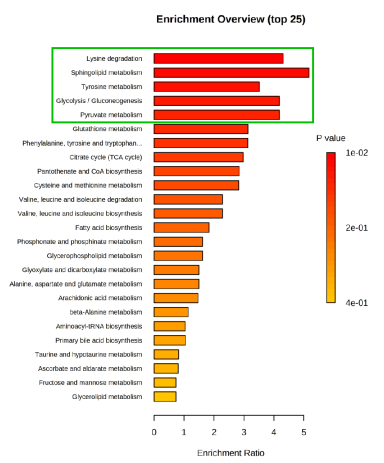

AT1: CON vs. TS+GA

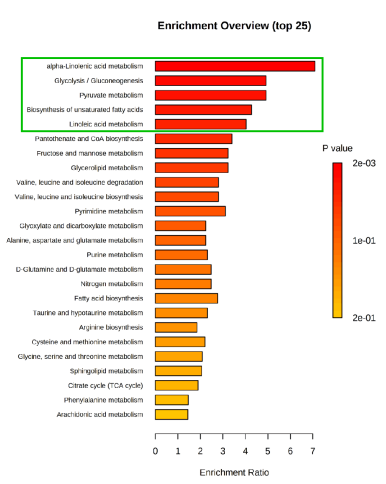

AT7: CON vs. TS+GA

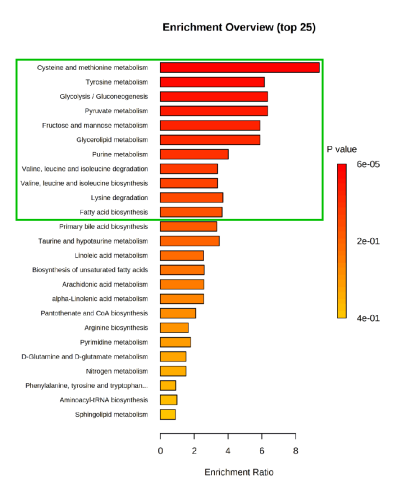

BT1: TS vs. TS+GA

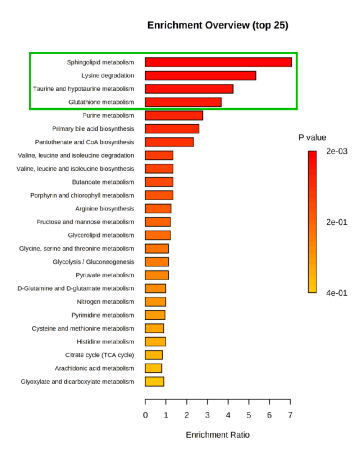

AT1: TS vs. TS+GA

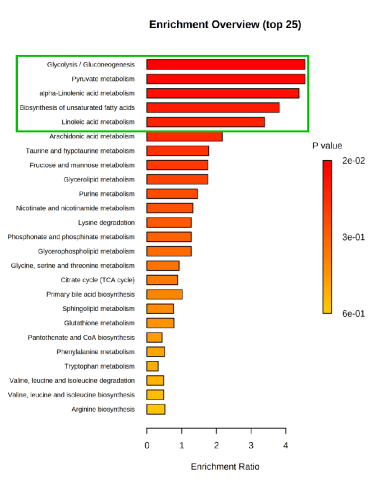

AT7: TS vs. TS+GA

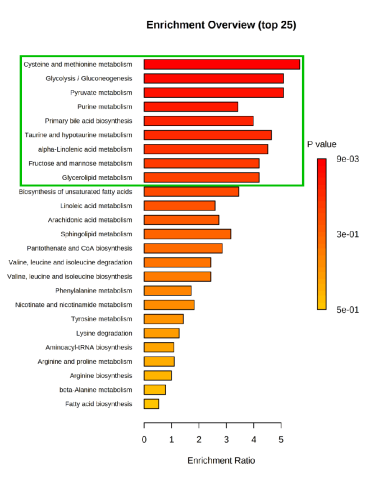

FIGURE 9 | Bar charts of the metabolic pathway analysis of differential serum metabolites on BT1 (A), AT1 (B), and AT7 (C) ( $\mathrm{n}=6$ or 7$)$. The pathway enrichment analysis shows all matched pathways, and the green boxes indicate significant metabolic pathways $(p<0.05)$. BT1, the 1st day before transportation; AT1, the 1st day after transportation; AT7, the 7th day after transportation.

dodecanoic acid and homovanillic acid were positively correlated with Eubacteriaceae.

\section{DISCUSSION}

To date, adequate evidence exists to support the antioxidation, anti-inflammatory, and antimicrobial activities of GA $(45,63$,
64). In this study, we summarized the results of $16 \mathrm{~S}$ rRNA gene sequencing and metabolomics analysis and discussed the effects of environmental stress and GA on the host-microbial metabolic axis from the relationship between metabolic biomarkers and gut bacteria. Our results suggested that dietary GA supplementation reduced multiple stress-induced diarrhea in puppies by enhancing systemic and intestinal defenses. The diarrhea rate still seemed to be high over a short period, and a possible reason 


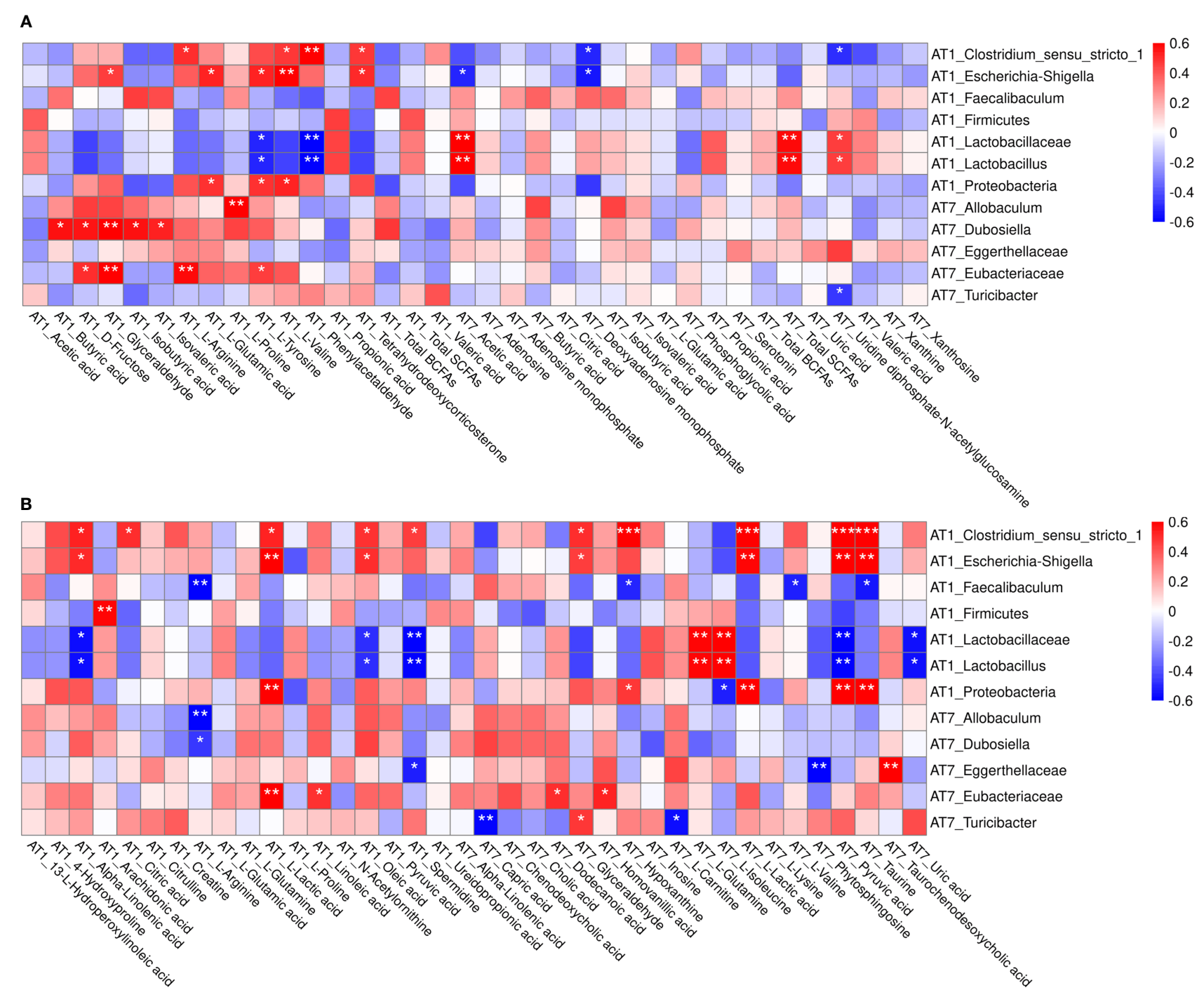

FIGURE 10 | Spearman's correlation analysis between the differential feces metabolites and fecal microbiota (A), and the differential serum metabolites and fecal microbiota (B) on AT1 and AT7. The symbol $\left(^{*}\right)$ indicates a significant association between metabolite and microbiota $\left({ }^{\star} P<0.05\right.$, ${ }^{\star \star} P<0.01$, and $\left.{ }^{\star \star \star} P<0.001\right)$. Red color indicates a positive correlation, and blue color indicates a negative correlation. AT1, the 1st day after transportation; AT7, the 7th day after transportation.

was the GM disturbance induced by stress, whereas puppies fed GA maintained normal FS and lower diarrhea rate by inhibiting the growth of pathogenic bacteria Escherichia-Shigella throughout the experiment. Unexpectedly, FS in the TS group fluctuated considerably before transportation, whose reason may be the large individual differences for all puppies in response to the stressful environment. A previous study conducted by Cai et al. (50) confirmed that dietary GA supplementation at $400 \mathrm{mg} /$ $\mathrm{kg}$ reduced diarrhea incidence in weaned piglets.

Meanwhile, stress activates the HPA axis and triggers a cascade of hormonal release $(65,66)$. Aligned with previous studies, the present study showed an increasing serum ACTH level after transportation, indicating that stress activates the hypothalamus to secrete $\mathrm{CRH}$ and induces the anterior pituitary gland to release
ACTH. The ACTH acts on the adrenal cortex to produce GC $\mathrm{COR}$, which negatively regulates $\mathrm{CRH}$ production to terminate the stress response cascade. However, dietary supplementation with $500 \mathrm{mg} / \mathrm{kg}$ of GA resulted in lower serum COR, GC, and ACTH levels on day 7 after transportation, indicating that GA has great potential to relieve stress. High HSP-70 level is also induced by inflammatory stress and oxidative stress except for heat shock (6769). Our results revealed that puppies in the TS group had higher HSP-70 levels after transportation, which were consistent with increased inflammatory response (TNF- $\alpha \uparrow, \mathrm{IL}-4 \downarrow)$ and oxidative stress $(\mathrm{GSH}-\mathrm{Px} \downarrow)$ caused by transportation and changing environment, while GA suppressed upregulation of HSP-70 level. Similarly, studies on other polyphenol compounds in animals also obtained similar results $(70,71)$. 
Previous studies revealed that the addition of dietary GA could modulate different signaling pathways through a wide range of inflammatory cytokines and enzymatic and nonenzymatic antioxidant defense systems (72). The enzymatic antioxidant defense system is generally the primary line of antioxidant defense in ROS detoxification (73). Additionally, MDA is the principal end-product of the lipid peroxidation process (74). So far, several studies reported the antioxidant action of GA $(64,75)$, and GA could provide the protection for various potential diseases including cancer, cardiovascular disease, and metabolic disease under oxidative stress by restoring the lipid peroxidation levels, normalizing or enhancing the levels of SOD, CAT, GSH-Px, GST, and GSH (76-78). In the present study, we found that multiple stressors resulted in a significant decrease in serum GSH-Px activities and an increase in MDA production in puppies. Nevertheless, dietary supplementation with GA at $500 \mathrm{mg} / \mathrm{kg}$ protected puppies from oxidative damage by increasing the activity of serum GSH-Px, which can efficiently eliminate free radicals and reduce the synthesis of MDA. Our results were consistent with those described in other studies.

Cytokines also play an important role in the regulation of intestinal function (79), while the overproduction of proinflammatory cytokines has a negative influence on intestinal homeostasis (80). It has been reported that the release of the pro-/anti-inflammatory and inflammatory mediators, such as IL-2, IL-4, IL-5, IL-13, IL-33, TNF- $\alpha$, IFN$\gamma$, and NF- $\kappa B$, could be downregulated by GA to prevent excessive inflammatory responses (41, 81, 82). Similarly, our results indicated that environmental stress caused a systemic inflammatory response by decreasing serum IgG content, increasing the production of proinflammatory cytokines TNF$\alpha$ and IFN- $\gamma$, and reducing the secretion of anti-inflammatory cytokine IL- 4 contents. However, GA effectively reversed the inflammatory responses in puppies, indicating that $500 \mathrm{mg} / \mathrm{kg}$ of dietary GA can improve anti-inflammatory function in stressed puppies. Also, a recent review concluded that GA plays an antiinflammatory role by modulating the GM (40).

Previous studies indicated that GA was effective in a broad spectrum of antibacterial applications against pathogens including E. coli, Staphylococcus aureus, Pseudomonas aeruginosa, Klebsiella pneumonia, Streptococcus mutans, Chromobacterium violaceum, Campylobacter jejuni, and Listeria monocytogenes (83-85). Our study also reached a similar inhibitory effect on pathogenic bacteria. Dietary supplementation with $500 \mathrm{mg} / \mathrm{kg}$ of GA improved the bacterial diversity, inhibited the growth of Escherichia-Shigella and Clostridium_sensu_stricto_1, and enhanced Lactobacillus and Faecalibaculum, especially 1 day after transportation. Our results were further verified with LEfSe analysis, which also found that the TS group was associated with enrichment of Proteobacteria, Escherichia-Shigella, and E. coli, while Lactobacillus, L. murinus, and L. reuteri dominated the GA treatment $(86,87)$. Spearman's correlation analysis revealed the symbiotic relationship between bacteria. The high coexistence of Escherichia-Shigella with Streptococcus and Clostridium_ sensu_stricto_1 suggested the possibility of a syntrophic relationship among these bacteria. However, Escherichia-Shigella negatively correlated with Faecalibaculum, Lactobacillus, and Bifidobacterium. In agreement, the high relative abundance of pathogen Escherichia-Shigella is reported to be accompanied by the low relative abundance of Lactobacillus $(88,89)$. Our results are in general agreement with the previous studies, which found a decrease in Lactobacillaceae and Prevotellaceae and an increase in Firmicutes and Proteobacteria phyla in dextran sodium sulfateinduced colitis in mice, and GA treatment could modulate the microbiota composition toward a similar proportion to the control group $(45,90)$. Furthermore, Lima et al. reported that EscherichiaShigella is one of the leading pathogenic causes of diarrhea, affecting approximately 80-165 million individuals (91). We can therefore infer that GA has a potential prophylactic effect on diarrhea caused by Escherichia-Shigella. GA also can induce changes in the microbiota toward a more favorable composition and activity, including the production of SCFAs and BCFAs in the colon (90).

The digestive tract contains an abundance of gut microbiotaderived metabolites (92). As one of the most important microbiotaderived metabolites, SCFAs are generated through colonic fermentation of dietary fibers $(93,94)$ and exert a beneficial effect on host health by reducing colonic $\mathrm{pH}$ and inflammation $(95,96)$, stimulating enterocyte growth, and improving mucus production and epithelial health (97). A previous study showed that increases in fecal SCFAs were found when relative abundances of Firmicutes, Lactobacillaceae, Clostridiales, Roseburia, Lachnospiraceae, and Erysipelotrichaceae were increased (98). These studies were in accordance with our findings that dietary GA treatment led to the increment of fecal total SCFAs and acetic acid concentrations. Further Spearman's correlation analysis revealed that fecal SCFAs have a positive association with Firmicutes (Erysipelotrichaceae, Faecalibaculum, Allobaculum, Turicibacter, and Dubosiella) and Lactobacillaceae (Lactobacillus) after transportation. Fecal BCFAs (e.g., isobutyric, isovaleric acid, valeric acid) are generated by microbial fermentation of branched amino acids, valine, leucine, and isoleucine $(99,100)$ and have effects on lipid and glucose metabolism (101). The highest total BCFAs and isovaleric acid concentrations were observed in the TS+GA group at 1 day after transportation, which had a positive association with Faecalibaculum, indicating that BCFAs may be produced by Faecalibaculum. The conclusion needs further validation. In short, these results indicate that GA protects against environmental stressinduced inflammation by improving the intestinal microbial structure and increasing the relative abundance of SCFAproducing bacteria.

Microbiota-derived metabolites, often secreted in the intestine and translocated across the intestinal barrier into the circulating system, are very important modulators for host metabolism (102, 103). In our study, metabolomics based on UPLC-Orbitrap-MS/ MS analysis method was applied to investigate the changes of fecal metabolites in beagle dogs. The KEGG enrichment analysis declared that environmental stress mainly disturbed amino acid metabolism, carbohydrate metabolism, lipid metabolism, and metabolism of cofactors and vitamins in puppies, while dietary intake of GA helped to restore this imbalance. Changes in the 
metabolic pathway were consistent with the PICRUSt analysis. Our findings were largely similar to the results reported by the previous study, whose metabolic data revealed that the GAinduced feces and urine metabolic changes in mice mainly focus on increasing carbohydrate metabolism (gluco-related metabolism) and lipid metabolism (bile acid metabolism) and decreasing amino acid metabolism (45). By screening differential metabolites in major differential metabolic pathways, fecal phenylacetaldehyde, L-tyrosine, L-valine, serotonin (amino acid metabolism), xanthine, adenosine, xanthosine, uric acid, phosphoglycolic acid (carbohydrate metabolism), tetrahydrodeoxycorticosterone, and glyceraldehyde (lipid metabolism) were upregulated due to the GA treatment. We considered them as the biomarkers for evaluating the influence of dietary GA treatment on fecal metabolites in puppies.

4-O-Methygallic acid (4-OMeGA) is the primary metabolite of GA in human plasma and urine (104-106). The current study detected high levels of 4-OMeGA in the serum of puppies, indicating that GA may exert its function mainly by further transforming to 4-OMeGA. Serum metabolomics revealed that environmental stress mainly influenced amino acid metabolism, carbohydrate metabolism, lipid metabolism, energy metabolism, and nucleotide metabolism, while puppies fed GA reversed the shift. This finding is similar to that of Shi et al. who reported that metabolic changes associated with GA intake include glycogenolysis, glycolysis, tricarboxylic acid (TCA) cycle, and metabolism of nucleotides, choline, bile acids, amino acids (107). Consistent with fecal biomarkers analysis, serum metabolites of L-arginine, creatine, spermidine, 4-hydroxyproline, L-proline, L-glutamic acid, pyruvic acid, $\mathrm{N}$-acetylornithine, citrulline, L-glutamine, L-valine, L-isoleucine, L-lysine, carnitine (amino acid metabolism), citric acid, L-lactic acid, glyceraldehyde (carbohydrate metabolism), alpha-linolenic acid, oleic acid, linoleic acid, arachidonic acid, 13-L-hydroperoxylinoleic acid, chenodeoxycholic acid, taurine, cholic acid, taurochenodeoxycholic acid (lipid metabolism), ureidopropionic acid, hypoxanthine, inosine, and uric acid (nucleotide metabolism) were chosen as the biomarkers for evaluating the influence of dietary GA treatment on serum metabolites in puppies.

Spearman's correlation analysis found that fecal L-valine, Ltyrosine, L-glutamic acid, phenylacetaldehyde, and tetra hydrodeoxycorticosterone were positively correlated with the relative abundance of Clostridium_sensu_stricto_1 (Firmicutes) and Escherichia-Shigella (Proteobacteria). However, interestingly, Ltyrosine and phenylacetaldehyde were oppositely correlated with and Lactobacillus (Lactobacillaceae). Serum L-lactic acid, alphalinolenic acid, citric acid, oleic acid, and spermidine were positively correlated with Clostridium_sensu_stricto_1 and Escherichia-Shigella, whereas alpha-linolenic acid, oleic acid, and spermidine were negatively correlated with Lactobacillus (Lactobacillaceae). Simultaneously, the positive correlation between serum metabolites and bacteria were L-arginine (Faecalibaculum), phytosphingosine and taurochenodeoxycholic acid (Eggerthellaceae), L-carnitine (Turicibacter), and dodecanoic acid and homovanillic acid (Eubacteriaceae); and serum arachidonic acid and glyceraldehyde had a positive association with Firmicutes and Turicibacter, respectively. Further research is needed to provide a clear explanation between GM and fecal and serum metabolome in puppies supplemented with GA.

\section{CONCLUSION}

The GA markedly reduced the incidence of diarrhea and alleviated multiple environmental stressor-induced oxidative stress and inflammatory responses in puppies. The microbiome and metabolomics analyses revealed that environmental stress caused intestinal microbiota and metabolic disorders, while GA reversed the abnormalities. The comprehensive microbiota and metabolite relationships were established. In summary, we systematically elucidated the beneficial effects of GA treatment on stressed dogs from the host-microbial metabolic axis point of view. Future studies that can focus on the interactions between microbiota and metabolites may prove efficacious for understanding the precise mechanisms of the beneficial effects of polyphenol on health.

\section{DATA AVAILABILITY STATEMENT}

The datasets presented in this study can be found in online repositories. The names of the repository/repositories and accession number(s) can be found below: https://www.ncbi. nlm.nih.gov/bioproject/PRJNA782241.

\section{ETHICS STATEMENT}

The animal study was reviewed and approved by the Experimental Animal Ethics Committee of South China Agricultural University.

\section{AUTHOR CONTRIBUTIONS}

KY generated the ideas, designed the study, detected the samples, and wrote the initial manuscript. LinZ and BD guided and revised the manuscript. $\mathrm{XD}$ and $\mathrm{SJ}$ participated in the data analysis and contributed to the draft of the manuscript. JD made feasible suggestions for the experimental design and manuscript. $\mathrm{MZ}$ analyzed the results. CW, ZQX, LimZ, AT, SY, PL, ZLX, SH, and FZ detected the samples. All authors contributed to the article and approved the submitted version.

\section{FUNDING}

This project was supported by the National Natural Science Foundation of China (Grant Nos. 31790411 and 32002186), Natural Science Foundation of Guangdong Province (Grant No. 2020A1515010322), Guangdong Basic and Applied Basic Research 
Foundation (2019B1515210002), and Independent Research and Development Projects of Maoming Laboratory (2021ZZ003).

\section{ACKNOWLEDGMENTS}

We gratefully appreciate the Laboratory Animal Center at the South China Agricultural University (Guangzhou, China) and National Canine Laboratory Animal Resource Bank, Guangzhou

\section{REFERENCES}

1. Karl JP, Hatch AM, Arcidiacono SM, Pearce SC, Pantoja-Feliciano IG, Doherty LA, et al. Effects of Psychological, Environmental and Physical Stressors on the Gut Microbiota. Front Microbiol (2018) 9:2013. doi: $10.3389 /$ fmicb. 2018.02013

2. Gollwitzer ES, Marsland BJ. Impact of Early-Life Exposures on Immune Maturation and Susceptibility to Disease. Trends Immunol (2015) 36:68496. doi: 10.1016/j.it.2015.09.009

3. Mueller NT, Bakacs E, Combellick J, Grigoryan Z, Dominguez-Bello MG. The Infant Microbiome Development: Mom Matters. Trends Mol Med (2015) 21:109-17. doi: 10.1016/j.molmed.2014.12.002

4. Ralevski A, Horvath TL. Developmental Programming of Hypothalamic Neuroendocrine Systems. Front Neuroendocrin (2015) 39:52-8. doi: 10.1016/j.yfrne.2015.09.002

5. Cain DW, Cidlowski JA. Immune Regulation by Glucocorticoids. Nat Rev Immunol (2017) 17:233-47. doi: 10.1038/nri.2017.1

6. Treccani G, Musazzi L, Perego C, Milanese M, Nava N, Bonifacino T, et al. Stress and Corticosterone Increase the Readily Releasable Pool of Glutamate Vesicles in Synaptic Terminals of Prefrontal and Frontal Cortex. Mol Psychiatry (2014) 19:433-43. doi: 10.1038/mp.2014.5

7. Adinoff B, Junghanns K, Kiefer F, Krishnan-Sarin S. Suppression of the HPA Axis Stress-Response: Implications for Relapse. Alcohol Clin Exp Res (2005) 29:1351-55. doi: 10.1097/01.alc.0000176356.97620.84

8. Hoffman KW, Lee JJ, Corcoran CM, Kimhy D, Kranz TM, Malaspina D. Considering the Microbiome in Stress-Related and Neurodevelopmental Trajectories to Schizophrenia. Front Psychiatry (2020) 11:629. doi: 10.3389/ fpsyt.2020.00629

9. Abbas Z, Hu L, Fang H, Sammad A, Kang L, Brito LF, et al. Association Analysis of Polymorphisms in the 5' Flanking Region of the HSP70 Gene With Blood Biochemical Parameters of Lactating Holstein Cows Under Heat and Cold Stress. Anim (Basel) (2020) 10:2016. doi: 10.3390/ani10112016

10. Katikaridis P, Bohl V, Mogk A. Resisting the Heat: Bacterial Disaggregases Rescue Cells From Devastating Protein Aggregation. Front Mol Biosci (2021) 8:681439. doi: 10.3389/fmolb.2021.681439

11. Li X, Yu Y, Gorshkov B, Haigh S, Bordan Z, Weintraub D, et al. Hsp70 Suppresses Mitochondrial Reactive Oxygen Species and Preserves Pulmonary Microvascular Barrier Integrity Following Exposure to Bacterial Toxins. Front Immunol (2018) 9:1309. doi: 10.3389/fimmu.2018.01309

12. Richter K, Haslbeck M, Buchner J. The Heat Shock Response: Life on the Verge of Death. Mol Cell (2010) 40:253-66. doi: 10.1016/j.molcel.2010.10.006

13. Liedel JL, Guo Y, Yu Y, Shiou S, Chen S, Petrof EO, et al. Mother's MilkInduced Hsp70 Expression Preserves Intestinal Epithelial Barrier Function in an Immature Rat Pup Model. Pediatr Res (2011) 69:395-400. doi: 10.1203/PDR.0b013e3182114ec9

14. Kojima K, Musch MW, Ren H, Boone DL, Hendrickson BA, Ma A, et al. Enteric Flora and Lymphocyte-Derived Cytokines Determine Expression of Heat Shock Proteins in Mouse Colonic Epithelial Cells. Gastroenterology (2003) 124:1395-407. doi: 10.1016/S0016-5085(03)00215-4

15. Herzog F, Loza K, Balog S, Clift MJD, Epple M, Gehr P, et al. Mimicking Exposures to Acute and Lifetime Concentrations of Inhaled Silver Nanoparticles by Two Different In Vitro Approaches. Beilstein J Nanotech (2014) 5:1357-70. doi: 10.3762/bjnano.5.149

16. Medzhitov R. Origin and Physiological Roles of Inflammation. Nature (2008) 454:428-35. doi: 10.1038/nature07201
General Pharmaceutical Research Institute Co., Ltd (Guangzhou, China), for providing the experimental sites.

\section{SUPPLEMENTARY MATERIAL}

The Supplementary Material for this article can be found online at: https://www.frontiersin.org/articles/10.3389/fimmu.2021.813890/ full\#supplementary-material

17. Marin IA, Goertz JE, Ren T, Rich SS, Onengut-Gumuscu S, Farber E, et al Microbiota Alteration is Associated With the Development of StressInduced Despair Behavior. Sci Rep (2017) 7:43859. doi: 10.1038/srep43859

18. Galley JD, Nelson MC, Yu Z, Dowd SE, Walter J, Kumar PS, et al. Exposure to a Social Stressor Disrupts the Community Structure of the Colonic Mucosa-Associated Microbiota. BMC Microbiol (2014) 14:189. doi: 10.1186/1471-2180-14-189

19. Bailey MT. Influence of Stressor-Induced Nervous System Activation on the Intestinal Microbiota and the Importance for Immunomodulation. Adv Exp Med Biol (2014) 817:255-76. doi: 10.1007/978-1-4939-0897-4_12

20. Zhou X, Johnson JS, Spakowicz D, Zhou W, Zhou Y, Sodergren E, et al. Longitudinal Analysis of Serum Cytokine Levels and Gut Microbial Abundance Links IL-17/IL-22 With Clostridia and Insulin Sensitivity in Humans. Diabetes (2020) 69:1833-42. doi: 10.2337/db19-0592

21. Pickard JM, Zeng MY, Caruso R, Núñez G. Gut Microbiota: Role in Pathogen Colonization, Immune Responses, and Inflammatory Disease. Immunol Rev (2017) 279:70-89. doi: 10.1111/imr.12567

22. Sánchez-Tapia M, Miller AW, Granados-Portillo O, Tovar AR, Torres N. The Development of Metabolic Endotoxemia is Dependent on the Type of Sweetener and the Presence of Saturated Fat in the Diet. Gut Microbes (2020) 12:1801301. doi: 10.1080/19490976.2020.1801301

23. Hill C, Guarner F, Reid G, Gibson GR, Merenstein DJ, Pot B, et al. The International Scientific Association for Probiotics and Prebiotics Consensus Statement on the Scope and Appropriate Use of the Term Probiotic. Nat Rev Gastro Hepat (2014) 11:506-14. doi: 10.1038/nrgastro.2014.66

24. Lomax AR, Calder PC. Prebiotics, Immune Function, Infection and Inflammation: A Review of the Evidence. Brit J Nutr (2009) 101:633-58. doi: $10.1017 /$ S0007114508055608

25. Machiels K, Joossens M, Sabino J, De Preter V, Arijs I, Eeckhaut V, et al. A Decrease of the Butyrate-Producing Species Roseburia Hominis and Faecalibacterium Prausnitzii Defines Dysbiosis in Patients With Ulcerative Colitis. Gut (2014) 63:1275-83. doi: 10.1136/gutjnl-2013-304833

26. Sokol H, Pigneur B, Watterlot L, Lakhdari O, Bermudez-Humaran LG, Gratadoux JJ, et al. Faecalibacterium Prausnitzii is an Anti-Inflammatory Commensal Bacterium Identified by Gut Microbiota Analysis of Crohn Disease Patients. P Natl Acad Sci USA (2008) 105:16731-36. doi: 10.1073/ pnas.0804812105

27. Lloyd-Price J, Arze C, Ananthakrishnan AN, Schirmer M, Avila-Pacheco J, Poon TW, et al. Multi-Omics of the Gut Microbial Ecosystem in Inflammatory Bowel Diseases. Nature (2019) 569:655-62. doi: 10.1038/ s41586-019-1237-9

28. Louis P, Hold GL, Flint HJ. The Gut Microbiota, Bacterial Metabolites and Colorectal Cancer. Nat Rev Microbiol (2014) 12:661-72. doi: 10.1038/nrmicro3344

29. Geuking MB, McCoy KD, Macpherson AJ. Metabolites From Intestinal Microbes Shape Treg. Cell Res (2013) 23:1339-40. doi: 10.1038/cr.2013.125

30. Arpaia N, Campbell C, Fan X, Dikiy S, van der Veeken J, DeRoos P, et al. Metabolites Produced by Commensal Bacteria Promote Peripheral Regulatory T-Cell Generation. Nature (2013) 504:451-55. doi: 10.1038/ nature 12726

31. Furusawa Y, Obata Y, Fukuda S, Endo TA, Nakato G, Takahashi D, et al. Commensal Microbe-Derived Butyrate Induces the Differentiation of Colonic Regulatory T Cells. Nature (2013) 504:446-50. doi: 10.1038/nature12721

32. Clemente JC, Manasson J, Scher JU. The Role of the Gut Microbiome in Systemic Inflammatory Disease. Bmj-Brit Med J (2018) 360:j5145. doi: $10.1136 / \mathrm{bmj} . j 5145$ 
33. Garrett WS, Gallini CA, Yatsunenko T, Michaud M, DuBois A, Delaney ML, et al. Enterobacteriaceae Act in Concert With the Gut Microbiota to Induce Spontaneous and Maternally Transmitted Colitis. Cell Host Microbe (2010) 8:292-300. doi: 10.1016/j.chom.2010.08.004

34. Pandey KB, Rizvi SI. Plant Polyphenols as Dietary Antioxidants in Human Health and Disease. Oxid Med Cell Longev (2009) 2:270-78. doi: 10.4161/ oxim.2.5.9498

35. Vezza T, Rodriguez-Nogales A, Algieri F, Utrilla MP, Rodriguez-Cabezas ME, Galvez J. Flavonoids in Inflammatory Bowel Disease: A Review. Nutrients (2016) 8:211. doi: 10.3390/nu8040211

36. Wang K, Jin X, Li Q, Sawaya ACHF, Le Leu RK, Conlon MA, et al. Propolis From Different Geographic Origins Decreases Intestinal Inflammation and Bacteroides Spp. Populations in a Model of DSS-Induced Colitis. Mol Nutr Food Res (2018) 62:1800080. doi: 10.1002/mnfr.201800080

37. Tomas-Barberan FA, Selma MV, Espin JC. Interactions of Gut Microbiota With Dietary Polyphenols and Consequences to Human Health. Curr Opin Clin Nutr (2016) 19:471-76. doi: 10.1097/MCO.0000000000000314

38. Cory H, Passarelli S, Szeto J, Tamez M, Mattei J. The Role of Polyphenols in Human Health and Food Systems: A Mini-Review. Front Nutr (2018) 5:87. doi: 10.3389/fnut.2018.00087

39. Wang K, Wan Z, Ou A, Liang X, Guo X, Zhang Z, et al. Monofloral Honey From a Medical Plant, Prunella Vulgaris, Protected Against Dextran Sulfate Sodium-Induced Ulcerative Colitis via Modulating Gut Microbial Populations in Rats. Food Funct (2019) 10:3828-38. doi: 10.1039/C9FO00460B

40. Yang K, Zhang L, Liao P, Xiao Z, Zhang F, Sindaye D, et al. Impact of Gallic Acid on Gut Health: Focus on the Gut Microbiome, Immune Response, and Mechanisms of Action. Front Immunol (2020) 11:580208. doi: 10.3389/ fimmu.2020.580208

41. Hyun KH, Gil KC, Kim SG, Park SY, Hwang KW. Delphinidin Chloride and its Hydrolytic Metabolite Gallic Acid Promote Differentiation of Regulatory T Cells and Have an Anti-Inflammatory Effect on the Allograft Model. J Food Sci (2019) 84:920-30. doi: 10.1111/1750-3841.14490

42. BenSaad LA, Kim KH, Quah CC, Kim WR, Shahimi M. Anti-Inflammatory Potential of Ellagic Acid, Gallic Acid and Punicalagin a\&B Isolated From Punica Granatum. BMC Complement Altern Med (2017) 17:47. doi: 10.1186/ s12906-017-1555-0

43. Phonsatta N, Deetae P, Luangpituksa P, Grajeda-Iglesias C, FigueroaEspinoza MC, Le Comte J, et al. Comparison of Antioxidant Evaluation Assays for Investigating Antioxidative Activity of Gallic Acid and its Alkyl Esters in Different Food Matrices. J Agr Food Chem (2017) 65:7509-18. doi: $10.1021 /$ acs.jafc.7b02503

44. Wang Y, Xie M, Ma G, Fang Y, Yang W, Ma N, et al. The Antioxidant and Antimicrobial Activities of Different Phenolic Acids Grafted Onto Chitosan. Carbohyd Polym (2019) 225:115238. doi: 10.1016/j.carbpol.2019.115238

45. Li Y, Xie Z, Gao T, Li L, Chen Y, Xiao D, et al. A Holistic View of Gallic AcidInduced Attenuation in Colitis Based on Microbiome-Metabolomics Analysis. Food Funct (2019) 10:4046-61. doi: 10.1039/c9fo00213h

46. Coelho LP, Kultima JR, Costea PI, Fournier C, Pan Y, Czarnecki-Maulden G, et al. Similarity of the Dog and Human Gut Microbiomes in Gene Content and Response to Diet. Microbiome (2018) 6:72. doi: 10.1186/s40168-018-0450-3

47. Laflamme D. Development and Validation of a Body Condition Score System for Dogs. Canine Pract (1997) 22:10-5. doi: 10.2307/1592173

48. Association of American Feed Control Officials (AAFCO). Official Publication. Oxford, IN: AAFCO (2017).

49. National Research Council. Nutrient Requirements of Dogs and Cats. Washington, DC: National Research Council of the National Academies (2006).

50. Cai L, Li YP, Wei ZX, Li XL, Jiang XR. Effects of Dietary Gallic Acid on Growth Performance, Diarrhea Incidence, Intestinal Morphology, Plasma Antioxidant Indices, and Immune Response in Weaned Piglets. Anim Feed Sci Tech (2020) 261:114391. doi: 10.1016/j.anifeedsci.2020.114391

51. AOAC. Official Methods of Analysis. Association of Official Analytical Chemists Publ. 17th. Gaithersburg (MD: AOAC International (2000).

52. Middelbos IS, Fastinger NDJr., Fahey GC. Evaluation of Fermentable Oligosaccharides in Diets Fed to Dogs in Comparison to Fiber Standards. J Anim Sci (2007) 85:3033-44. doi: 10.2527/jas.2007-0080

53. Magoc T, Salzberg SL. FLASH: Fast Length Adjustment of Short Reads to Improve Genome Assemblies. Bioinformatics (2011) 27:2957-63. doi: 10.1093/bioinformatics/btr507
54. Bokulich NA, Subramanian S, Faith JJ, Gevers D, Gordon JI, Knight R, et al. Quality-Filtering Vastly Improves Diversity Estimates From Illumina Amplicon Sequencing. Nat Methods (2013) 10:57-9. doi: 10.1038/nmeth.2276

55. Caporaso JG, Kuczynski J, Stombaugh J, Bittinger K, Bushman FD, Costello EK, et al. QIIME Allows Analysis of High-Throughput Community Sequencing Data. Nat Methods (2010) 7:335-36. doi: 10.1038/nmeth.f.303

56. Edgar RC, Haas BJ, Clemente JC, Quince C, Knight R. UCHIME Improves Sensitivity and Speed of Chimera Detection. Bioinformatics (2011) 27:2194200. doi: 10.1093/bioinformatics/btr381

57. Haas BJ, Gevers D, Earl AM, Feldgarden M, Ward DV, Giannoukos G, et al. Chimeric 16s rRNA Sequence Formation and Detection in Sanger and 454Pyrosequenced PCR Amplicons. Genome Res (2011) 21:494-504. doi: $10.1101 /$ gr.112730.110

58. Edgar RC. UPARSE: Highly Accurate OTU Sequences From Microbial Amplicon Reads. Nat Methods (2013) 10:996-98. doi: 10.1038/nmeth.2604

59. Quast C, Pruesse E, Yilmaz P, Gerken J, Schweer T, Yarza P, et al. The SILVA Ribosomal RNA Gene Database Project: Improved Data Processing and WebBased Tools. Nucleic Acids Res (2013) 41:D590-96. doi: 10.1093/nar/gks1219

60. Edgar RC. MUSCLE: Multiple Sequence Alignment With High Accuracy and High Throughput. Nucleic Acids Res (2004) 32:1792-97. doi: 10.1093/ nar/gkh340

61. Nuli R, Azhati J, Cai J, Kadeer A, Zhang B, Mohemaiti P. Metagenomics and Faecal Metabolomics Integrative Analysis Towards the Impaired Glucose Regulation and Type 2 Diabetes in Uyghur-Related Omics. J Diabetes Res (2019) 2019:2893041. doi: 10.1155/2019/2893041

62. Xin Z, Ma S, Ren D, Liu W, Han B, Zhang Y, et al. UPLC-Orbitrap-MS/MS Combined With Chemometrics Establishes Variations in Chemical Components in Green Tea From Yunnan and Hunan Origins. Food Chem (2018) 266:534-44. doi: 10.1016/j.foodchem.2018.06.056

63. Sohrabi F, Dianat M, Badavi M, Radan M, Mard SA. Gallic Acid Suppresses Inflammation and Oxidative Stress Through Modulating Nrf2-HO-1-NF- kb Signaling Pathways in Elastase-Induced Emphysema in Rats. Environ Sci Pollut R (2021) 28:56822-34. doi: 10.1007/s11356-021-14513-1

64. Dludla PV, Nkambule BB, Jack B, Mkandla Z, Mutize T, Silvestri S, et al. Inflammation and Oxidative Stress in an Obese State and the Protective Effects of Gallic Acid. Nutrients (2019) 11:23. doi: 10.3390/nu11010023

65. McEWEN BS. Stress, Adaptation, and Disease: Allostasis and Allostatic Load. Ann Ny Acad Sci (1998) 840:33-44. doi: 10.1111/j.1749-6632.1998. tb09546.x

66. Cohen S, Janicki-Deverts D, Miller GE. Psychological Stress and Disease. Jama-J Am Med Assoc (2007) 298:1685. doi: 10.1001/jama.298.14.1685

67. Dastoor Z, Dreyer J. Nuclear Translocation and Aggregate Formation of Heat Shock Cognate Protein 70 (Hsc70) in Oxidative Stress and Apoptosis. J Cell Sci (2000) 113:2845-54. doi: 10.1242/jcs.113.16.2845

68. Sevin M, Girodon F, Garrido C, de Thonel A. HSP90 and HSP70: Implication in Inflammation Processes and Therapeutic Approaches for Myeloproliferative Neoplasms. Mediat Inflamm (2015) 2015:1-08. doi: 10.1155/2015/970242

69. Huo C, Xiao C, She R, Liu T, Tian J, Dong H, et al. Chronic Heat Stress Negatively Affects the Immune Functions of Both Spleens and Intestinal Mucosal System in Pigs Through the Inhibition of Apoptosis. Microb Pathogenesis (2019) 136:103672. doi: 10.1016/j.micpath.2019.103672

70. Hu H, Bai X, Xu K, Zhang C, Chen L. Effect of Phloretin on Growth Performance, Serum Biochemical Parameters and Antioxidant Profile in Heat-Stressed Broilers. Poultry Sci (2021) 100:101217. doi: 10.1016/ j.ps.2021.101217

71. Venuprasad MP, Kandikattu HK, Razack S, Amruta N, Khanum F. Chemical Composition of Ocimum Sanctum by LC-ESI-MS/MS Analysis and its Protective Effects Against Smoke Induced Lung and Neuronal Tissue Damage in Rats. BioMed Pharmacother (2017) 91:1-12. doi: 10.1016/ j.biopha.2017.04.011

72. Gao J, Hu J, Hu D, Yang X. A Role of Gallic Acid in Oxidative Damage Diseases: A Comprehensive Review. Nat Prod Commun (2019) 14:1-09. doi: $10.1177 / 1934578 X 19874174$

73. Tan H, Wang N, Li S, Hong M, Wang X, Feng Y. The Reactive Oxygen Species in Macrophage Polarization: Reflecting its Dual Role in Progression and Treatment of Human Diseases. Oxid Med Cell Longev (2016) 2016:1-16. doi: $10.1155 / 2016 / 2795090$ 
74. Todorova I, Simeonova G, Kyuchukova D, Dinev D, Gadjeva V. Reference Values of Oxidative Stress Parameters (MDA, SOD, CAT) in Dogs and Cats. Comp Clin Path (2005) 13:190-94. doi: 10.1007/s00580-005-0547-5

75. Moradi A, Abolfathi M, Javadian M, Heidarian E, Roshanmehr H, Khaledi M, et al. Gallic Acid Exerts Nephroprotective, Anti-Oxidative Stress, and AntiInflammatory Effects Against Diclofenac-Induced Renal Injury in Malerats. Arch Med Res (2021) 52:380-88. doi: 10.1016/j.arcmed.2020.12.005

76. Moghtaderi H, Sepehri H, Delphi L, Attari F. Gallic Acid and Curcumin Induce Cytotoxicity and Apoptosis in Human Breast Cancer Cell MDA-MB231. BioImpacts (2018) 8:185-94. doi: 10.15171/bi.2018.21

77. Kang N, Lee J, Lee W, Ko J, Kim E, Kim J, et al. Gallic Acid Isolated From Spirogyra Sp. Improves Cardiovascular Disease Through a Vasorelaxant and Antihypertensive Effect. Environ Toxicol Phar (2015) 39:764-72. doi: 10.1016/ j.etap.2015.02.006

78. Hsu C, Yen G. Effect of Gallic Acid on High Fat Diet-Induced Dyslipidaemia, Hepatosteatosis and Oxidative Stress in Rats. Brit J Nutr (2007) 98:727-35. doi: 10.1017/S000711450774686X

79. Neurath MF. Cytokines in Inflammatory Bowel Disease. Nat Rev Immunol (2014) 14:329-42. doi: 10.1038/nri3661

80. Al-Sadi R. Mechanism of Cytokine Modulation of Epithelial Tight Junction Barrier. Front Biosci-Landmrk (2009) 14:2765-78. doi: 10.2741/3413

81. Radan M, Dianat M, Badavi M, Mard SA, Bayati V, Goudarzi G. In Vivo and In Vitro Evidence for the Involvement of Nrf2-Antioxidant Response Element Signaling Pathway in the Inflammation and Oxidative Stress Induced by Particulate Matter (PM10): The Effective Role of Gallic Acid. Free Radical Res (2019) 53:210-25. doi: 10.1080/10715762.2018.1563689

82. Wang X, Zhao H, Ma C, Lv L, Feng J, Han S. Gallic Acid Attenuates Allergic Airway Inflammation via Suppressed Interleukin-33 and Group 2 Innate Lymphoid Cells in Ovalbumin-Induced Asthma in Mice. Int Forum Allergy $R h$ (2018) 8:1284-90. doi: 10.1002/alr.22207

83. Shao D, Li J, Li J, Tang R, Liu L, Shi J, et al. Inhibition of Gallic Acid on the Growth and Biofilm Formation of Escherichia Coli and Streptococcus Mutans. J Food Sci (2015) 80:M1299-305. doi: 10.1111/1750-3841.12902

84. Sorrentino E, Succi M, Tipaldi L, Pannella G, Maiuro L, Sturchio M, et al. Antimicrobial Activity of Gallic Acid Against Food-Related Pseudomonas Strains and its Use as Biocontrol Tool to Improve the Shelf Life of Fresh Black Truffles. Int J Food Microbiol (2018) 266:183-89. doi: 10.1016/ j.ijfoodmicro.2017.11.026

85. Oh E, Jeon B. Synergistic Anti-Campylobacter Jejuni Activity of Fluoroquinolone and Macrolide Antibiotics With Phenolic Compounds. Front Microbiol (2015) 6:1129. doi: 10.3389/fmicb.2015.01129

86. Gong ZP, Ouyang J, Wu XL, Zhou F, Lu DM, Zhao CJ, et al. Dark Tea Extracts: Chemical Constituents and Modulatory Effect on Gastrointestinal Function. BioMed Pharmacother (2020) 130:110514. doi: 10.1016/ j.biopha.2020.110514

87. Pacheco-Ordaz R, Wall-Medrano A, Goñi MG, Ramos-Clamont-Montfort G, Ayala-Zavala JF, González-Aguilar GA. Effect of Phenolic Compounds on the Growth of Selected Probiotic and Pathogenic Bacteria. Lett Appl Microbiol (2018) 66:25-31. doi: 10.1111/lam.12814

88. Li Q, Peng X, Burrough ER, Sahin O, Gould SA, Gabler NK, et al. Dietary Soluble and Insoluble Fiber With or Without Enzymes Altered the Intestinal Microbiota in Weaned Pigs Challenged With Enterotoxigenic E. Coli F18. Front Microbiol (2020) 11:1110. doi: 10.3389/fmicb.2020.01110

89. Xu X, Wang H, Guo D, Man X, Liu J, Li J, et al. Curcumin Modulates Gut Microbiota and Improves Renal Function in Rats With Uric Acid Nephropathy. Renal Failure (2021) 43:1063-75. doi: 10.1080/0886022X. 2021.1944875

90. Pandurangan AK, Mohebali N, Mohd. Esa N, Looi CY, Ismail S, Saadatdoust Z. Gallic Acid Suppresses Inflammation in Dextran Sodium Sulfate-Induced Colitis in Mice: Possible Mechanisms. Int Immunopharmacol (2015) 28:1034-43. doi: 10.1016/j.intimp.2015.08.019

91. Lima IF, Havt A, Lima AA. Update on Molecular Epidemiology of Shigella Infection. Curr Opin Gastroenterol (2015) 31:30-7. doi: 10.1097/MOG. 0000000000000136

92. Chamorro S, Romero C, Brenes A, Sánchez-Patán F, Bartolomé B, Viveros A, et al. Impact of a Sustained Consumption of Grape Extract on Digestion, Gut Microbial Metabolism and Intestinal Barrier in Broiler Chickens. Food Funct (2019) 10:1444-54. doi: 10.1039/C8FO02465K
93. Borton MA, Sabag-Daigle A, Wu J, Solden LM, O’Banion BS, Daly RA, et al. Chemical and Pathogen-Induced Inflammation Disrupt the Murine Intestinal Microbiome. Microbiome (2017) 5:47. doi: 10.1186/s40168-017-0264-8

94. Woting A, Blaut M. The Intestinal Microbiota in Metabolic Disease. Nutrients (2016) 8:202. doi: 10.3390/nu8040202

95. Fei Y, Wang Y, Pang Y, Wang W, Zhu D, Xie M, et al. Xylooligosaccharide Modulates Gut Microbiota and Alleviates Colonic Inflammation Caused by High Fat Diet Induced Obesity. Front Physiol (2020) 10:1601. doi: 10.3389/ fphys.2019.01601

96. Sivaprakasam S, Prasad PD, Singh N. Benefits of Short-Chain Fatty Acids and Their Receptors in Inflammation and Carcinogenesis. Pharmacol Therapeut (2016) 164:144-51. doi: 10.1016/j.pharmthera.2016.04.007

97. Park J, Kotani T, Konno T, Setiawan J, Kitamura Y, Imada S, et al. Promotion of Intestinal Epithelial Cell Turnover by Commensal Bacteria: Role of Short-Chain Fatty Acids. PloS One (2016) 11:e156334. doi: 10.1371/ journal.pone. 0156334

98. Estaki M, Pither J, Baumeister P, Little JP, Gill SK, Ghosh S, et al. Cardiorespiratory Fitness as a Predictor of Intestinal Microbial Diversity and Distinct Metagenomic Functions. Microbiome (2016) 4:42. doi: 10.1186/s40168-016-0189-7

99. Lynch CJ, Adams SH. Branched-Chain Amino Acids in Metabolic Signalling and Insulin Resistance. Nat Rev Endocrinol (2014) 10:723-36. doi: 10.1038/ nrendo.2014.171

100. Yao CK, Muir JG, Gibson PR. Review Article: Insights Into Colonic Protein Fermentation, its Modulation and Potential Health Implications. Aliment Pharmacol Ther (2016) 43:181-96. doi: 10.1111/apt.13456

101. Heimann E, Nyman M, Pålbrink A, Lindkvist-Petersson K, Degerman E. Branched Short-Chain Fatty Acids Modulate Glucose and Lipid Metabolism in Primary Adipocytes. Adipocyte (2016) 5:359-68. doi: 10.1080/21623945.2016.1252011

102. Canfora EE, Meex RCR, Venema K, Blaak EE. Gut Microbial Metabolites in Obesity, NAFLD and T2DM. Nat Rev Endocrinol (2019) 15:261-73. doi: 10.1038/s41574-019-0156-z

103. Vítek L, Haluzík M. The Role of Bile Acids in Metabolic Regulation. J Endocrinol (2016) 228:R85-96. doi: 10.1530/JOE-15-0469

104. Ferruzzi MG, Lobo JK, Janle EM, Cooper B, Simon JE, Wu Q, et al. Bioavailability of Gallic Acid and Catechins From Grape Seed Polyphenol Extract is Improved by Repeated Dosing in Rats: Implications for Treatment in Alzheimer's Disease. J Alzheimers Dis (2009) 18:113-24. doi: 10.3233/JAD-2009-1135

105. Abd El Mohsen MM, Kuhnle G, Rechner AR, Schroeter H, Rose S, Jenner P, et al. Uptake and Metabolism of Epicatechin and its Access to the Brain After Oral Ingestion. Free Radical Bio Med (2002) 33:1693-702. doi: 10.1016/ S0891-5849(02)01137-1

106. Shahrzad S, Bitsch I. Determination of Gallic Acid and its Metabolites in Human Plasma and Urine by High-Performance Liquid Chromatography. J Chromatogr B (1998) 705:87-95. doi: 10.1016/S0378-4347(97)00487-8

107. Shi X, Xiao C, Wang Y, Tang H. Gallic Acid Intake Induces Alterations to Systems Metabolism in Rats. J Proteome Res (2013) 12:991-1006. doi: $10.1021 /$ pr301041k

Conflict of Interest: AT is employed by Guangzhou Qingke Biotechnology Co., Ltd.

The remaining authors declare that the research was conducted in the absence of any commercial or financial relationships that could be construed as a potential conflict of interest.

Publisher's Note: All claims expressed in this article are solely those of the authors and do not necessarily represent those of their affiliated organizations, or those of the publisher, the editors and the reviewers. Any product that may be evaluated in this article, or claim that may be made by its manufacturer, is not guaranteed or endorsed by the publisher.

Copyright $\odot 2022$ Yang, Deng, Jian, Zhang, Wen, Xin, Zhang, Tong, Ye, Liao, Xiao, $H e$, Zhang, Deng, Zhang and Deng. This is an open-access article distributed under the terms of the Creative Commons Attribution License (CC BY). The use, distribution or reproduction in other forums is permitted, provided the original author(s) and the copyright owner(s) are credited and that the original publication in this journal is cited, in accordance with accepted academic practice. No use, distribution or reproduction is permitted which does not comply with these terms. 